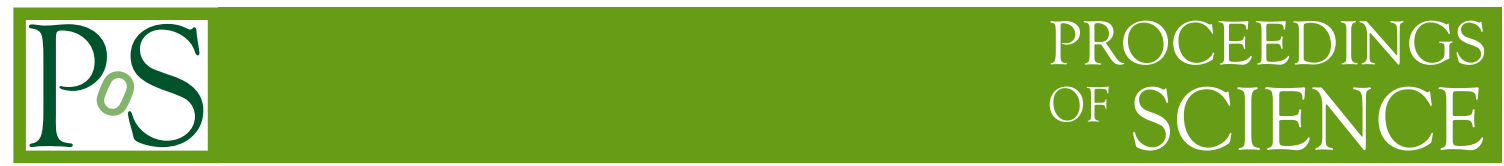

\title{
Higgs Physics in ATLAS
}

\author{
Dimitris Fassouliotis, on behalf of the ATLAS collaboration* \\ Univeristy of Athens \\ Physics Faculty, Panepistimioupoli, Ilissia, Greece \\ E-mail: Dimitrios.Fassouliotis@cern.ch
}

\begin{abstract}
An overview of the Higgs boson physics program performed in the ATLAS experiment at LHC is presented, using the entire set of Run I data collected in 2011 and 2012. Emphasis is given to the studies that followed the discovery of the new boson, aiming to investigate its properties. No indications for deviations from the Standard Model nature of the Higgs boson are observed. Searches for additional Higgs bosons are also briefly reported.
\end{abstract}

Proceedings of the Corfu Summer Institute 2014 "School and Workshops on Elementary Particle Physics and Gravity",

3-21 September 2014

Corfu, Greece

${ }^{*}$ Speaker. 


\section{Introduction}

One of the primary goals of the physics programme of the Large Hadron Collider (LHC) [1], is the exploration of the electro-weak symmetry breaking mechanism and the study of mass generation for the elementay particles. In the Standard Model (SM) of elementary particles this is achieved through the Englert-Brout-Higgs mechanism [2,3]. An immediate consequence of this mechanism is the existence of the Higgs boson, which was the only SM particle that was not observed before the start of proton-proton collisions at LHC.

LHC has been operated in 2011 and 2012 at center-of-mass energies $\sqrt{s}$ of 7 and $8 \mathrm{TeV}$, respectively. On 4th July 2012 both the ATLAS and CMS Collaborations [4,5], having analyzed each $\sim 10 \mathrm{fb}^{-1}$ of data, announced the discovery of a Higgs-like particle with mass around $125 \mathrm{GeV}$. The corresponding publications of September $2012[6,7]$ reported the discovery of a new boson with a significance of equal or above $5.0 \sigma$ for both experiments. The mass of the Higgs boson is not predicted by the SM. However, once its mass is determined, all the rest of the properties of the Higgs boson are defined in SM and therefore their precise measurement is of vital importance. Later, as more data were collected, the properties (spin, CP, couplings) of the discovered particle were measured and in March 2013 CERN dropped the word "like" from the name of the new boson.

The ATLAS detector has performed very efficiently during Run I, having collected data corresponding to an integrated luminosity of around $26 \mathrm{fb}^{-1}\left(\sim 5 \mathrm{fb}^{-1}\right.$ at $\sqrt{s}=7 \mathrm{TeV}$ and $\sim 21 \mathrm{fb}^{-1}$ at $\sqrt{s}=8 \mathrm{TeV}$ ). This report summarizes recent experimental results by ATLAS on the search of a SM-like Higgs boson, the determination of its properties and searches for Higgs bosons beyond the SM.

\section{Higgs boson production and decays at LHC}

At the LHC, the dominant production mode at the Higgs boson mass of $125 \mathrm{GeV}$, is through the gluon-gluon fusion (ggF) mechanism, where gluons inside the protons interact indirectly mainly via a top-quark loop with the Higgs boson. This mechanism accounts for the $87 \%$ of the total production. Figure 1(a) shows the SM Higgs boson production cross sections for $p p$ collisions at $\sqrt{s}=8 \mathrm{TeV}$ for the various productions mechanisms as a function of the Higgs mass [8]. The other mechanisms, vector boson fusion (VBF), the associated production with a $W$ or $Z$ boson $(\mathrm{VH})$ and the associated production with a pair of top quarks $(\mathrm{ttH})$ are at the few $\%$ level. However, the decay products of the $W$ and $Z$ bosons or top quarks as well as the additional forward jets in the VBF production, are extremely useful to distinguish the SM Higgs boson signal from background processes in certain decay channels and to study its couplings through the measurement of the rate of the different production modes. Figure 1(b) shows the SM Higgs boson decay branching ratios at $\sqrt{s}=8 \mathrm{TeV}$ as a function of the Higgs mass. It is important to indicate that certain decay modes (like $H \rightarrow b \bar{b}$ or $H \rightarrow \tau^{+} \tau^{-}$, which have large branching ratios, suffer from huge backgrounds and can only be distinguished through production modes with additional particles in the final state. The SM Higgs boson production cross sections at different energies have been calculated by theory including the most recent higher order corrections and are tabulated in [8] as a function of the Higgs boson mass. 


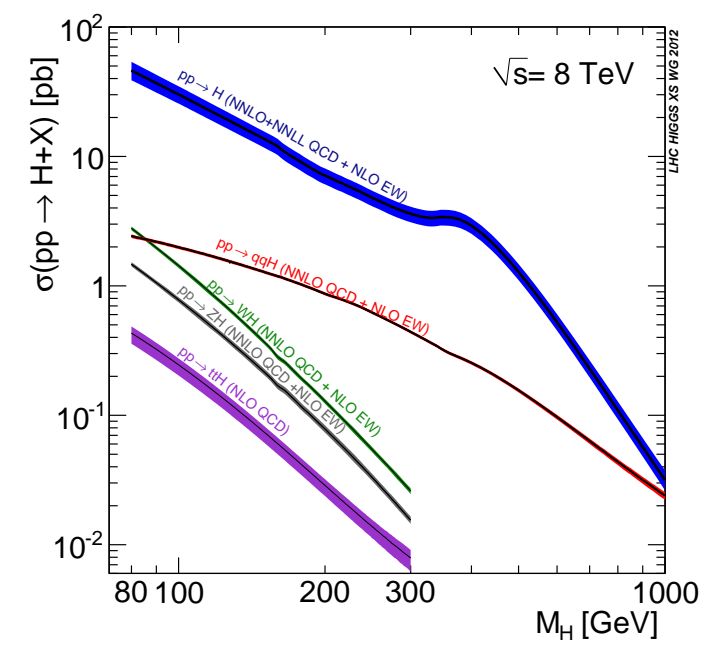

(a)

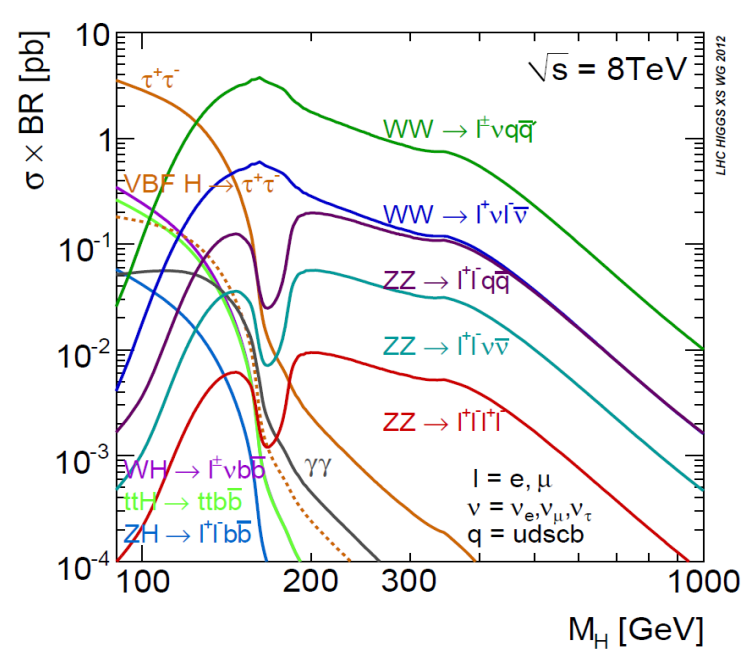

(b)

Figure 1: (a) SM Higgs boson production cross sections for $p p$ collisions at $\sqrt{s}=8 \mathrm{TeV}$ and (b) Higgs branching fractions as a function of the Higgs boson mass [8].

\section{The ATLAS detector}

The ATLAS detector [4] is a multipurpose particle detector with approximately forwardbackward cylindrically symmetric geometry. The inner tracking detector (ID) consists of a silicon pixel detector, which is closest to the interaction point, and a silicon microstrip detector surrounding the pixel detector, both covering a pseudorapidity range $|\eta|<2.5$, followed by a transition radiation straw-tube tracker (TRT) covering $|\eta|<2$. The ID is surrounded by a thin superconducting solenoid providing a $2 \mathrm{~T}$ axial magnetic field. A highly segmented lead/liquid argon (LAr) sampling electromagnetic calorimeter measures the energy and the position of electromagnetic showers with $|\eta|<3.2$. The LAr calorimeter includes a presampler (for $|\eta|<1.8$ ) and three sampling layers, longitudinal in shower depth, for $|\eta|<2.5$. LAr sampling calorimeters are also used to measure hadronic showers in the endcaps $(1.5<|\eta|<3.2)$ and electromagnetic and hadronic showers in the forward $(3.1<|\eta|<4.9)$ regions, while an iron/scintillator tile calorimeter measures hadronic showers in the central region $(|\eta|<1.7)$. The muon spectrometer (MS) surrounds the calorimeters and is designed to detect muons in the pseudorapidity range up to $|\eta|=2.7$. The MS consists of one barrel $(|\eta|=1.05)$ and two endcap parts. A system of three large superconducting air-core toroid magnets, each with eight coils, provides a magnetic field with a bending integral of about $2.5 \mathrm{~T} \cdot \mathrm{m}$ in the barrel and up to $6 \mathrm{~T} \cdot \mathrm{m}$ in the endcaps. Monitored drift-tube chambers in both the barrel and endcap regions and cathode strip chambers covering $|\eta|>2$ are used as precision chambers, whereas resistive plate chambers in the barrel and thin gap chambers in the endcaps are used as trigger chambers, covering up to $|\eta|=2.4$. The chambers are arranged in three layers, so high-pT particles traverse at least three stations with a lever arm of several meters. A three-level trigger system selects events to be recorded for offline analysis. 


\section{Physics Object Definition}

Electrons and photons are reconstructed using information from the ID and the electromagnetic calorimeter. For electrons, background discrimination relies on the shower shape information available from the highly segmented LAr electromagnetic calorimeter, high-threshold TRT hits, as well as compatibility of the tracking and calorimeter information. Muons are reconstructed as tracks in the ID and MS, and their identification is primarily based on the presence of a matching track or tag in the MS. Jets are reconstructed from clusters of calorimeter cells and calibrated using a dedicated scheme designed to adjust the energy measured in the calorimeter to that of the true jet energy.

\subsection{Electron and photon reconstruction and idenitification}

Electrons and photons are reconstructed from clusters of energy deposits in the electromagnetic calorimeter [9], [10]. Tracks matched to electron candidates (and, for $8 \mathrm{TeV}$ data, from photon conversions) and having enough associated hits in the silicon detectors are fitted using a GaussianSum Filter (GSF), which accounts for bremsstrahlung energy loss [11]. The GSF fit reduces the difference between the energy measured in the calorimeter and the momentum measured in the ID and improves the measured electron direction and impact parameter resolutions. The electron transverse energy is computed from the cluster energy and the track direction at the interaction point. Electron candidates are reconstructed in the pseudorapidity interval $|\eta|<2.47$, and are required to have a well-reconstructed ID track pointing to the electromagnetic calorimeter cluster. The cluster should satisfy a set of identification criteria that require the longitudinal and transverse shower profiles to be consistent with those expected for electromagnetic showers [9]. The electron identification was improved for the 2012 data set by moving from a cut-based method to a likelihood method. The likelihood allows the inclusion of discriminating variables that are difficult to use with explicit cuts without incurring significant efficiency losses.

Photon candidates are required to have a transverse energy greater than $15 \mathrm{GeV}$ and pseudorapidity within the regions $|\eta|<1.37$ or $1.52<|\eta|<2.37$, where the first calorimeter layer has high granularity. Electromagnetic clusters reconstructed in or near regions of the calorimeter affected by read-out or high voltage failures are not accepted. The identification of photons is performed through a cut-based selection based on shower shapes measured in the first two longitudinal layers of the electromagnetic calorimeter and on the leakage into the hadronic calorimeter [12].

Recent improvements of the calibration strategy for the energy measurement of electrons and photons is described in references $[10,13]$. To achieve the best energy resolution and to minimize systematic uncertainties, the calibration and stability of the calorimeter cell energy measurement is optimized, the relative calibration of the longitudinal layers of the calorimeter is adjusted and a determination of the amount of material in front of the calorimeter is performed. The global calorimeter energy scale is then determined in situ with a large sample of $Z \rightarrow e^{+} e^{-}$events, and verified using $J / \psi \rightarrow e^{+} e^{-}$and $Z \rightarrow \ell^{+} \ell^{-} \gamma$ events, showing good agreement with the energy scale determined from the $Z \rightarrow e^{+} e^{-}$sample. Compared to the previous studies, the uncertainties in the calibration are significantly reduced by using data-driven measurements for the intercalibration of the calorimeter layers and for the estimate of the material in front of the calorimeter, as well as by improving the accuracy of the in situ calibration with $Z \rightarrow e^{+} e^{-}$events. The calibration 


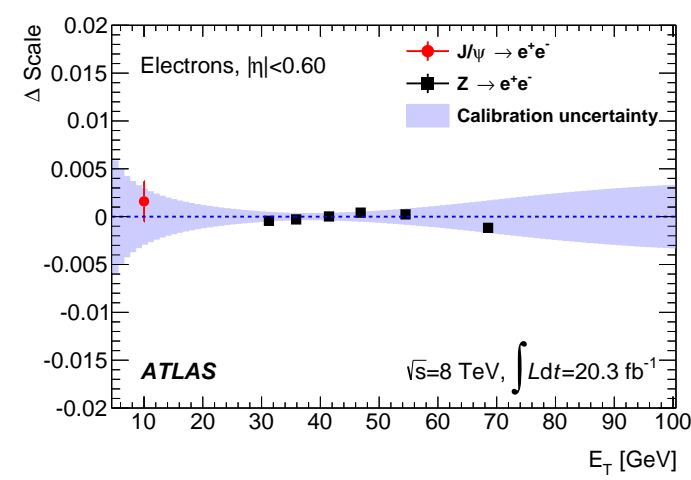

(a)

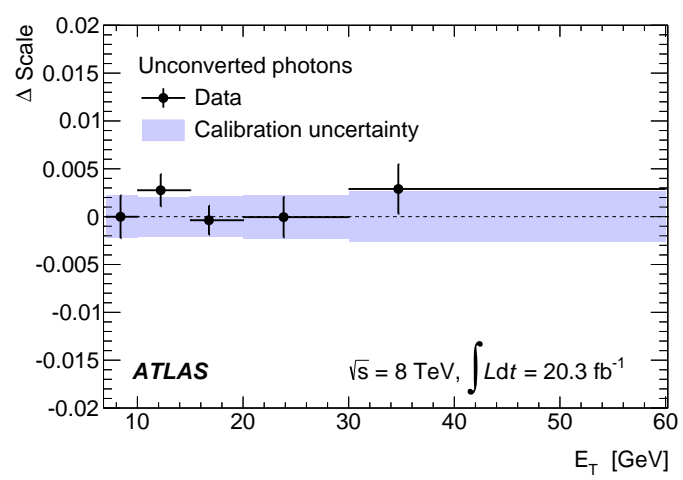

(b)

Figure 2: (a) Relative scale difference ( $\Delta$ Scale) between the measured electron energy scale and the nominal energy scale, as a function of $E_{\mathrm{T}}$ using $Z \rightarrow e^{+} e^{-}$and $J / \psi \rightarrow e^{+} e^{-}$events (points with error bars), for $|\eta|<0.6$. The uncertainty on the nominal energy scale for electrons is shown as the shaded area. The error bars include the systematic uncertainties specific to the $J / \psi \rightarrow e^{+} e^{-}$measurement. (b) Relative scale difference between the measured photon energy scale using $Z \rightarrow \ell^{+} \ell^{-} \gamma$ events and the nominal energy scale as a function of $E_{\mathrm{T}}$ for unconverted photons. The $Z \rightarrow \ell^{+} \ell^{-} \gamma$ measurements are the points with error bars. The uncertainty on the nominal energy scale for photons is shown as the shaded area [13].

analysis, using a total of 6.6 million $Z \rightarrow e^{+} e^{-}$events, determines the scale for electrons with transverse energy $E_{\mathrm{T}} \sim 40 \mathrm{GeV}$. Any systematic uncertainty has thus minimal impact on these electrons, but can lead to residual non-linearities and differences between the electron, unconverted photon and converted photon energy scales, which were carefully studied. The uncertainty on the electron energy scale is in general less than $0.1 \%$, and up to $0.3 \%$ for $|\eta| \sim 1.5$ at the barrel/endcap transition. At $E_{\mathrm{T}}$ of about $60 \mathrm{GeV}$, the total uncertainty on the photon energy scale is between $0.2 \%$ and $0.3 \%$ and up to $0.9 \%$ and $0.4 \%$ in the transition region, for unconverted and converted photons, respectively. Figure 2 presents examples of the verification of the electron and photon energy scales from these samples after the full calibration procedure is applied.

\subsection{Muon reconstruction and idenitification}

Four types of muon candidates are distinguished, depending on how they are reconstructed. Most muon candidates are identified by matching a reconstructed ID track with either a complete or partial track reconstructed in the MS [14]. If a complete MS track is present, the two independent momentum measurements are combined (combined muons), otherwise the momentum is measured using the ID, and the partial MS track serves as identification (segment-tagged muons). The muon reconstruction and identification coverage is extended by using tracks reconstructed in the forward region $(2.5<|\eta|<2.7)$ of the MS, which is outside the ID coverage (standalone muons). In the center of the barrel region $(|\eta|<0.1)$, which lacks MS geometrical coverage, ID tracks with $p_{\mathrm{T}}>15 \mathrm{GeV}$ are identified as muons if their calorimetric energy deposition is consistent with a minimum ionizing particle (calorimeter-tagged muons). The inner detector tracks associated with muons that are identified inside the ID acceptance are required to have a minimum number of associated hits in each of the ID subdetectors to ensure good track reconstruction. The muon 
candidates outside the ID acceptance that are reconstructed only in the MS, are required to have hits in each of the three stations they traverse. In the analyses described below, at most one standalone or calorimeter-tagged muon is used per event.

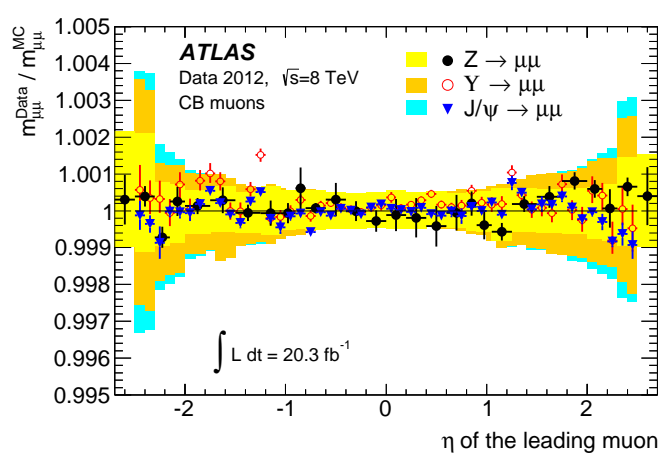

(a)

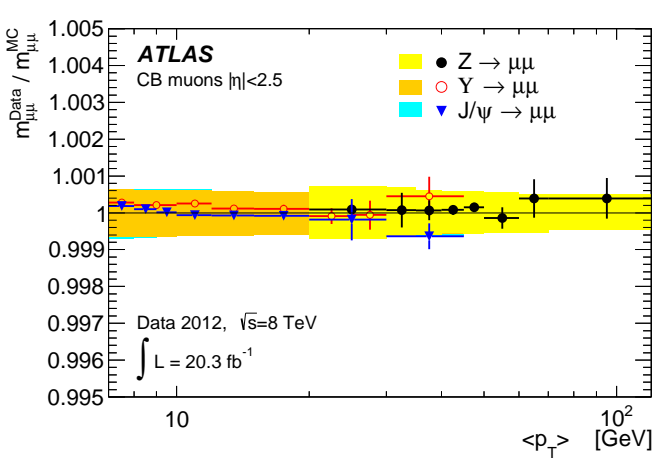

(b)

Figure 3: Ratio of the reconstructed dimuon invariant mass for data to the nominal mass in simulation for $J / \psi, \Upsilon$ and $Z$ events: (a) as a function of $\eta$ of the higher- $p_{\mathrm{T}}$ muon, and (b) as a function of the transverse momentum of the two muons. The shaded areas show the systematic uncertainty on the simulation corrections for each of the three samples. The error bars on the points show the combined statistical and systematic uncertainties [13].

Recent improvements have been applied on the muon momentum scale and resolution in simulation measured from collision data as described in detail in references [13,14]. About 6 million $J / \psi \rightarrow \mu^{+} \mu^{-}$events and about 9 million $Z \rightarrow \mu^{+} \mu^{-}$events were used to extract the corrections to be applied to the simulated data. They consist of scale corrections for the ID and MS, which were derived separately, a $p_{\mathrm{T}}$-independent momentum correction for the MS and a $p_{\mathrm{T}}$-dependent smearing correction to be applied in order to reproduce the resolution observed in data. The major improvement with respect to the previous studies, is the use of $J / \psi \rightarrow \mu^{+} \mu^{-}$events in addition to the $Z \rightarrow \mu^{+} \mu^{-}$sample in the simulation correction procedure. This allows a significant reduction of the momentum scale uncertainty in the low momentum range that is relevant for the $H \rightarrow Z Z^{*} \rightarrow 4 \ell$ mass measurement. The systematic uncertainties on the momentum scale of combined muons are $0.04 \%$ in the barrel region and increase to about $0.2 \%$ for $|\eta|>2$. These results were checked by separately fitting the dimuon invariant mass distribution to extract the peak position and the width of the $J / \psi, Z$ and $\Upsilon$ resonances in data and in the simulation, with and without corrections. For this study 17 million $J / \psi$ events were used. The $\Upsilon$ sample, of about 5 million events, was not used in the simulation correction procedure and therefore provides an independent validation. Figure 3(a) shows the ratio of the reconstructed dimuon invariant mass for data to the nominal mass (after the application of all corrections) in simulation for $J / \psi, \Upsilon$ and $Z$ events as a function of $\eta$ of the higher- $p_{\mathrm{T}}$ muon. Figure 3(b) shows the same ratio as a function of the average transverse momentum of the two muons. The error bars on data points show the combined statistical and systematic uncertainty. These studies demonstrate the validity of the corrections and of the associated systematic uncertainties in the range $6<p_{\mathrm{T}}<100 \mathrm{GeV}$. 


\subsection{Jet reconstruction}

Jets are reconstructed using the anti $-k_{t}$ algorithm [15] with a radius parameter $\mathrm{R}=0.4$. The inputs to the reconstruction are three-dimensional clusters of energy [16] in the calorimeter, calibrated to the electromagnetic energy scale and corrected for contributions from in-time and outof-time pile-up, and the position of the primary interaction vertex. The algorithm for this clustering suppresses noise by keeping only cells with a significant energy deposit and their neighboring cells. Subsequently, the jets are calibrated to the hadronic energy scale using $p_{\mathrm{T}}$ and $\eta$-dependent correction factors [16], [17]. The uncertainty on these correction factors is determined from control samples in data. To reduce the number of jet candidates originating from pile-up vertices, jets with $p_{\mathrm{T}}<50 \mathrm{GeV}$ within the ID acceptance $(|\eta|<2.4)$ are required to have more than $50 \%$ (75\% for 2011 data) of the summed scalar $p_{\mathrm{T}}$ of the tracks associated with the jet (within $\mathrm{R}=0.4$ around the jet axis) come from tracks of the primary vertex [18].

The identification of b-quark jets (b-jets) is limited to the acceptance of the ID $(|\eta|<2.5)$. The $b$-jets are identified using multivariate techniques [19] that are based on quantities that separate $b$ and $\mathrm{c}$ jets from light jets arising from light-flavor quarks and gluons. For an operation point of $80 \%$ efficiency, typical rejection factors are 26 and 1400 against c-jets and light jets, respectively [20].

\subsection{Tau lepton reconstruction and identification}

For leptonicaly decaying $\tau$ leptons, muons or electrons are identified as presented in the previous sections. The reconstruction of hadronically decaying tau leptons is seeded from jets. Tracks with $p_{\mathrm{T}}>1 \mathrm{GeV}$ within a cone of radius 0.2 around a cluster barycentre are matched to the $\tau_{\text {had }}$ candidate, and the charge of the candidate is determined from the sum of the charges of its associated tracks. The rejection of jets is provided using discriminating variables based on tracks with $p_{\mathrm{T}}>1 \mathrm{GeV}$ and the energy deposited in calorimeter cells found in the core region $(\Delta R<0.2)$ and in the region $0.2<\Delta R<0.4$ around the candidate's direction. Such discriminating variables are combined in a boosted decision tree (BDT) [21] and three working points are defined [22], corresponding to different identification efficiency values.

\subsection{Missing transverse momentum reconstruction}

The signature of a high-momentum neutrino is a momentum imbalance in the transverse plane. The missing transverse momentum $\left(E_{\mathrm{T}}^{\text {miss }}\right)$ is calculated [23] as the negative vector sum of the momentum of objects selected according to ATLAS identification algorithms, such as leptons, photons, and jets, and of the remaining "soft" objects that typically have low values of $p_{\mathrm{T}}$. The large coverage in rapidity $(y)$ of the calorimeter and its sensitivity to neutral particles motivate a calorimeter-based reconstruction of the missing transverse momentum. However, the significant pile-up present in the data degrades the resolution of the calorimeter-based measurement of missing transverse momentum. An improvement in resolution is obtained using a track-based measurement of the soft objects, where the tracks are required to have $p_{\mathrm{T}}>0.5 \mathrm{GeV}$ and originate from the primary vertex. Tracks associated with identified leptons or jets are not included, as these selected objects are added separately to the calculation of the missing transverse momentum. 


\section{Higgs Search in bosonic decay modes}

The bosonic decays were used as a tool for the discovery at the initial stage and subsequently as a tool for the measurement of the properties and the confirmation of the discovery. In all these channels the production is studied both inclusively and with events categorized according to the characteristics of the different production modes. The production rate relative to the SM expectation, the signal strength $(\mu)$, is determined using the full information from the categorized analyses.

\section{1 $H \rightarrow Z Z^{*} \rightarrow 4 \ell(\ell=e$ or $\mu)$ decay channel}

The $H \rightarrow Z Z^{*} \rightarrow 4 \ell$ channel has a small rate but produces a very clean final state with full reconstruction of the Higgs boson and signal to background ratio of about 1.5. It is therefore ideal for studies concerning the Higgs boson properties. The final state consists of four isolated leptons (electrons or muons) originating from the primary vertex, one pair of which is expected to have an invariant mass consistent with the $Z$ boson mass. The $Z Z^{*}$ continuum production is the irreducible background. The reducible background consists of $Z$ plus jets and $t \bar{t}$ production and it is estimated using data driven techniques, using isolation, impact parameter, reconstruction properties and the charge of the leptons to define appropriate control regions.

Single-lepton and dilepton triggers are used to select the four-lepton events with an efficiency, on the events that pass the final selection, which exceeds 97\%. Higgs boson candidates are formed by selecting two same-flavor, opposite-sign lepton pairs. Track and calorimetric isolation and impact parameter criteria are applied to all the lepton candidates, to ensure that they are prompt leptons originating from the primary vertex. Different momentum (energy) thresholds are applied to the leptons forming the quadruplet, with the minimum being $7 \mathrm{GeV}$ for muons and $6 \mathrm{GeV}$ for electrons. Events are categorized in subchannels according to the flavor composition of the pairs. A search is performed for final state radiation (FSR) photons [24] arising from any of the lepton candidates and at most one FSR photon candidate is added to the $4 \ell$ system. After the FSR correction, the lepton four-momenta of the lepton pair with the mass closest to the $Z$ boson mass are recomputed by means of a $Z$-mass-constrained kinematic fit. Apart from the above mentioned improvements, recent ATLAS results [25] exploit the new electron reconstruction and identification (see section 4.1) and take full advantage of the new calibration of the electromagnetic calorimeters and the new muon momentum scale studies (see section 4.2) to improve considerably the systematic uncertainties. Further categorization of the events, in order to distinguish the different production modes, proceeds according to the jet content of the events and the existence of additional leptons.

The analysis sensitivity is further improved by employing three multivariate discriminants to distinguish between the different classes of four-lepton events: one to separate the Higgs boson signal from the $Z^{*}$ background in the inclusive analysis, and two to separate the VBF- and VHproduced Higgs boson signal from the ggF produced Higgs boson signal in the VBF enriched and VH-hadronic enriched categories. These discriminants are based on BDTs. For the mass and the inclusive signal strength $(\mu)$ determination, a $2 \mathrm{D}$ fit is used based on four-lepton invariant mass $m_{4 l}$ and the $\mathrm{BDT}_{Z Z}$ response. Figure 4(a) shows the four-lepton invariant mass distribution of the candidate events that survive the criteria of the cut based inclusive analysis. The signal expectation shown is for a mass hypothesis of $m_{H}=125 \mathrm{GeV}$ and normalized to the inclusive fitted signal strength corresponding to the Higgs mass measurement of ATLAS (see section 7.1). The mass 
derived from this channel alone is estimated as $m_{H}=124.51 \pm 0.52$ (stat) \pm 0.06 (syst) GeV, with the main systematic uncertainty stemming from the muon (electron) momentum (energy) scale.

A fit to the categories using $m_{4 l}$ and the responses of the dedicated BDTs, provides estimations of the Higgs boson couplings. This fit can be constrained to extract a single overall signal strength for the $H \rightarrow Z Z^{*} \rightarrow 4 \ell$ final state. Figure 4(b) shows the $68 \%$ and $95 \%$ confidence level (CL) contours in the $\mu-m_{H}$ plane for the inclusive analysis. The combined measurement yields $\mu=1.44_{-0.31}^{+0.34}$ (stat) ${ }_{-0.11}^{+0.21}$ (syst) and the observed significance of the excess of events is $8.1 \sigma$ (with $6.2 \sigma$ expected). The dominant experimental uncertainty is due to the lepton reconstruction and identification efficiency, but it is much lower than the theoretical ones.

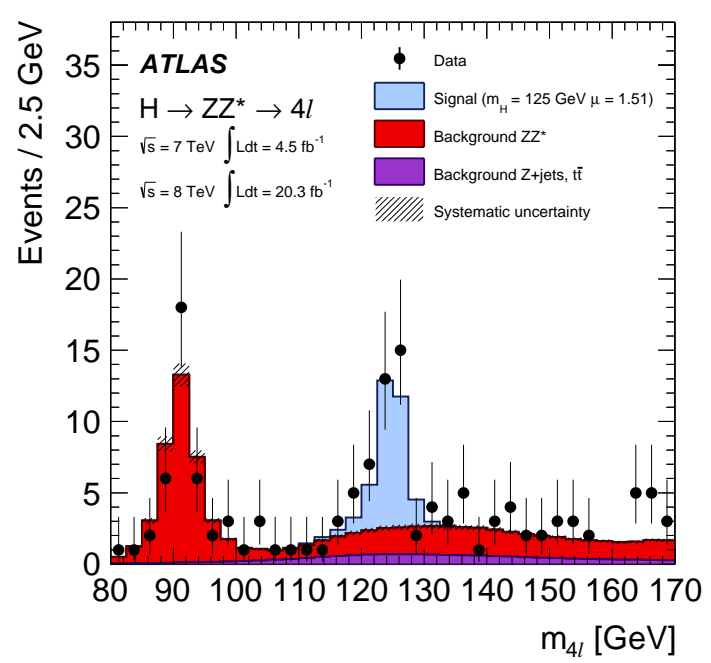

(a)

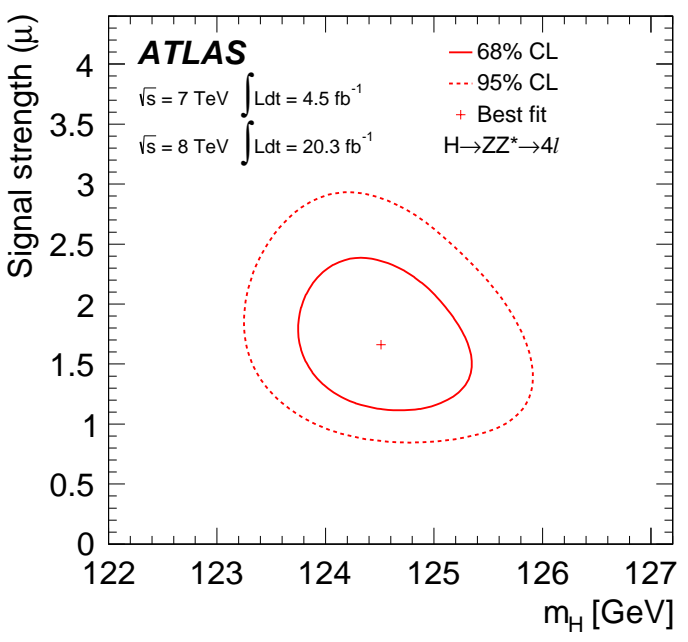

(b)

Figure 4: (a) The distribution of the four-lepton invariant mass, for the selected candidates (filled circles) compared to the expected signal and background contributions (filled histograms). The signal expectation shown is for a mass hypothesis of $m_{H}=125 \mathrm{GeV}$ and normalized to the inclusive fitted signal strength corresponding to the Higgs mass measurement of ATLAS. (b) The $68 \%$ and $95 \%$ confidence level (CL) contours in the $\mu-m_{H}$ plane for the inclusive $H \rightarrow Z Z^{*} \rightarrow 4 \ell$ analysis [25].

\section{$5.2 H \rightarrow \gamma \gamma$ decay channel}

The branching ratio of the $H \rightarrow \gamma \gamma$ channel is small, but it produces a clean final state with full reconstruction of the Higgs boson and a signal $m_{\gamma \gamma}$ peak on top of a large but smooth background distribution. It is therefore an excellent channel to measure the mass and couplings of the Higgs boson. The final state consists of two isolated photons originating from the primary vertex. The diphoton continuum production constitutes the dominant $(\sim 80 \%)$ irreducible background, while the reducible background consists of $\gamma$-jet and jet-jet. The invariant mass distributions and normalizations of the backgrounds are estimated by fits to control regions in the data, while the choices of the functional forms used to model the backgrounds and the uncertainties associated with these choices are determined mostly by MC studies. 
A diphoton trigger is used to select this final state with a signal efficiency above $99 \%$ for events fulfilling the final event selection. At least two photon candidates are required to be in the fiducial region of the EM calorimeter, excluding the transition region between the barrel and the end-cap calorimeters. The leading and sub-leading photon candidates are required to have $E_{\mathrm{T}} / m_{\gamma \gamma}>0.35$ and 0.25 , respectively. The diphoton production vertex is selected from the reconstructed collision vertices using a neural-network algorithm. This information is used for the accurate determination of the opening angle between the two photons, and the estimation of their isolation properties. A two-fold categorization of events is performed. First, categories aiming to distinguish events according to the production mechanism of the Higgs boson are formed (similarly to section 5.1). Furthermore, events are categorized according to the transverse momentum of the diphoton system and the pseudorapidity of the candidate photons.

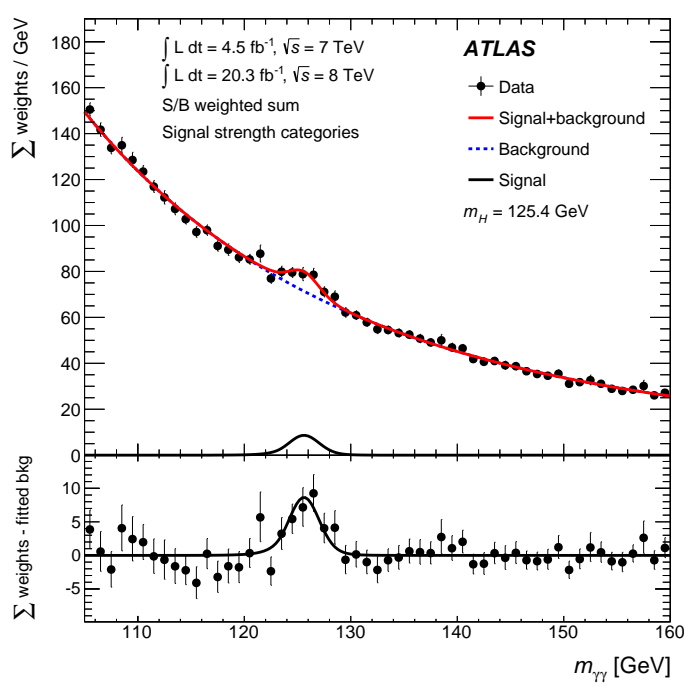

(a)

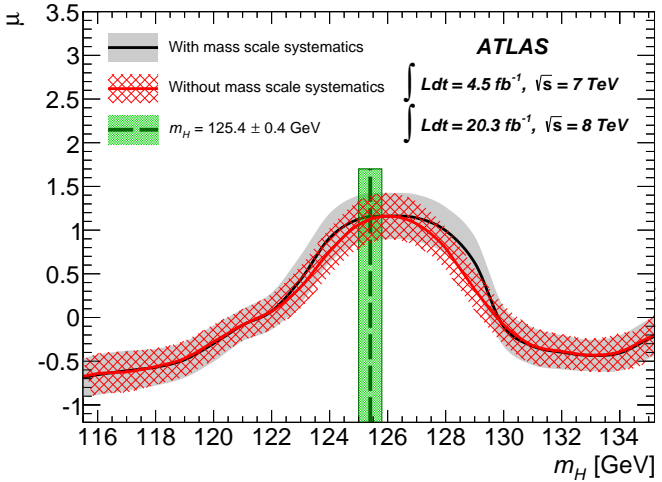

(b)

Figure 5: (a) Diphoton invariant mass $m_{\gamma \gamma}$ spectrum. Each event is weighted by the signal to background ratio in the dataset and category it belongs to. The solid red curve shows the fitted signal plus background model when the Higgs boson mass is fixed at $125.4 \mathrm{GeV}$. The background component of the fit is shown with the dotted blue curve. The signal component of the fit is shown with the solid black curve. The bottom plot shows the data relative to the background component of the fitted model. (b) The combined signal strength parameter $\mu$ versus $m_{H}$ with mass scale systematic uncertainties included (black curve) and excluded (red curve). The uncertainties on the measured $\mu$ are shown as gray (red) bands with the mass scale systematic uncertainties included (excluded). The vertical dotted line and shaded band indicate the value $m_{H}=125.4 \pm 0.4 \mathrm{GeV}[26]$.

In the final ATLAS analysis of Run-I data [26], the diphoton mass resolution is improved by $10 \%$ and the uncertainty on the photon energy resolution is reduced by approximately a factor of two with respect to the previous publications. The improvements stem from improvements on the detector simulation model, a better knowledge of the material upstream of the calorimeter, and more detailed calibration corrections applied to the data (see section 4.1). Figure 5(a) shows the diphoton invariant mass distribution of the candidate events weighted by the signal-to-background ratio in the dataset and category it belongs to. The solid red curve shows the fitted signal plus 
background model when the Higgs boson mass is fixed at $125.4 \mathrm{GeV}$. The background component of the fit is shown with the dotted blue curve. The signal component of the fit is shown with the solid black curve. Both the signal plus background and background-only curves reported here are obtained from the sum of the individual curves in each category weighted by their signal-tobackground ratio. The bottom plot shows the data relative to the background component of the fitted model. The mass derived from this channel alone is estimated as $m_{H}=125.98 \pm 0.42$ (stat) \pm 0.28 (syst) $\mathrm{GeV}$. The main systematic uncertainty of $0.22 \%$ is due to the photon energy scale.

An extended likelihood function is built from the number of observed events and analytic functions describing the distributions of $m_{\gamma \gamma}$ for the signal and the background in each event category. Individual as well as combined coupling measurements are derived from this likelihood. Figure 5(b) shows the combined signal strength parameter $\mu$ versus $m_{H}$. The combined signal strength for the $H \rightarrow \gamma \gamma$ final state is $\mu=1.17 \pm 0.23$ (stat) ${ }_{-0.08}^{+0.10}$ (syst) ${ }_{-0.08}^{+0.12}$ (theory), with the dominant experimental systematic uncertainty being from the photon energy resolution. The observed significance of the combined excess of events is $5.2 \sigma$ (with $4.6 \sigma$ expected).

\section{$5.3 H \rightarrow W W^{*} \rightarrow \ell v \ell v(\ell=e$ or $\mu)$ decay channel}

The $H \rightarrow W W^{*}$ decay mode has a large branching ratio. However, the cleaner way to observe this channel is through the final state $H \rightarrow W W^{*} \rightarrow \ell v \ell v$, where both the $W$ bosons decay leptonicaly to electrons or muons. Therefore, due to the two missing neutrinos the Higgs final state can not be reconstructed completely and the signal appears as a broad peak in the transverse mass $m_{\mathrm{T}}$ distribution. Depending on the production mechanism, the signal to background ratio ranges from 0.1 to 1 . The signal consists of two isolated, high $p_{\mathrm{T}}$, opposite sign leptons plus missing energy. The main irreducible background is the $W W\left(^{*}\right)$ continuum production, while many sources contribute to the reducible one, such as single top, $t \bar{t}, W / Z$ plus jets and other diboson production. For each background type the event selection includes a targeted set of requirements to distinguish the background from the signal. The background estimates are made with control regions in which some or all of these requirements are inverted.

Single-lepton and dilepton triggers are used to select the events with an efficiency which varies between $81 \%$ to $97 \%$ depending on the flavor composition and on the momentum of the leptons. The Higgs boson candidates are formed by selecting two opposite-sign leptons, with $p_{\mathrm{T}}$ thresholds of $22 \mathrm{GeV}$ for the leading lepton and $10 \mathrm{GeV}$ for the subleading one. Track and calorimetric isolation, as well as impact parameter, criteria are applied to both of them. Events are categorized in subchannels according to the flavor composition of the lepton pair and the number of reconstructed jets in the event. Further categorization of the events, to distinguish the VBF production mode, is performed using a BDT multivariate method. Depending on the category of the events, missing transverse energy criteria, as well as other kinematic requirements are applied to reduce considerably the backgrounds.

A likelihood function is constructed to simultaneously model the yields of the various subsamples in both the signal and the various control regions. To evaluate the signal strength parameter $\mu$, the likelihood is maximized profiling the scale factors which determine the background contributions as well as the rest of the systematic uncertainties. Figure 6(a) shows the post-fit combined transverse mass distributions for $n_{j} \leq 1$ for the three lepton-flavor samples. The bottom plot shows the residuals of the data with respect to the estimated background compared to the expected distri- 
bution for a SM Higgs boson with $m_{H}=125 \mathrm{GeV}$. The level of agreement observed between the background-subtracted data and the expected Higgs boson signal strengthens the interpretation of the observed excess as a signal from Higgs boson decay. The observed significance of the excess of events is $6.1 \sigma$ (with $5.8 \sigma$ expected). The assumption that the total yield is predicted by the SM is relaxed to evaluate the two-dimensional likelihood contours of $\left(m_{H}, \mu\right)$, shown in Figure 6(b). The final result of the Run I analysis, presented in [27], for the combined signal strength measurement for $H \rightarrow W W^{*} \rightarrow \ell v \ell v$ yields $\mu=1.09_{-0.15}^{+0.16}$ (stat) ${ }_{-0.14}^{+0.17}$ (syst).

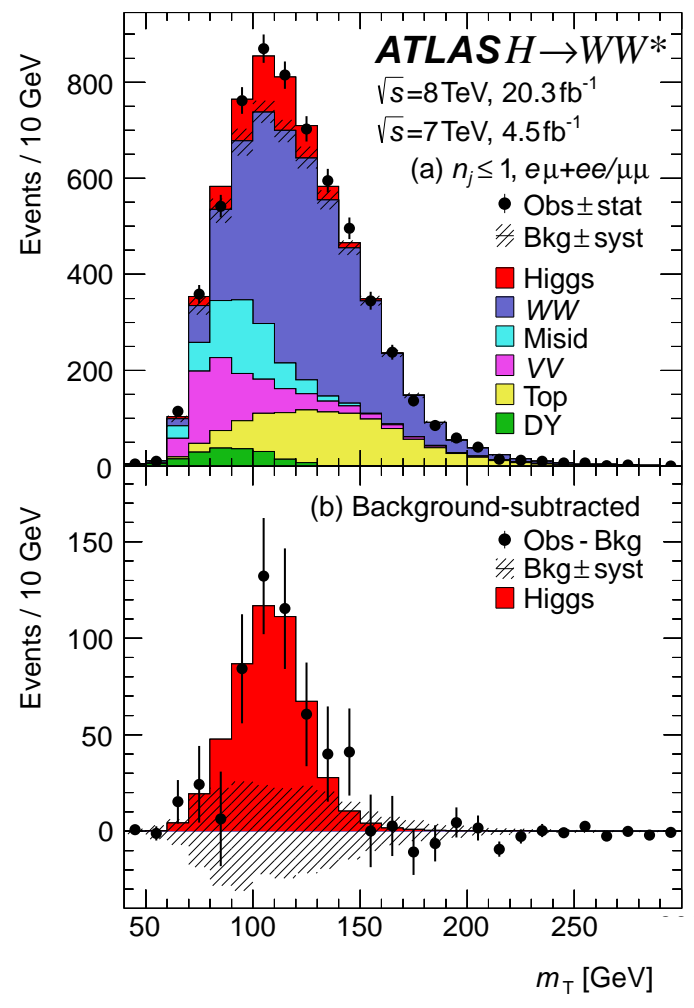

(a)

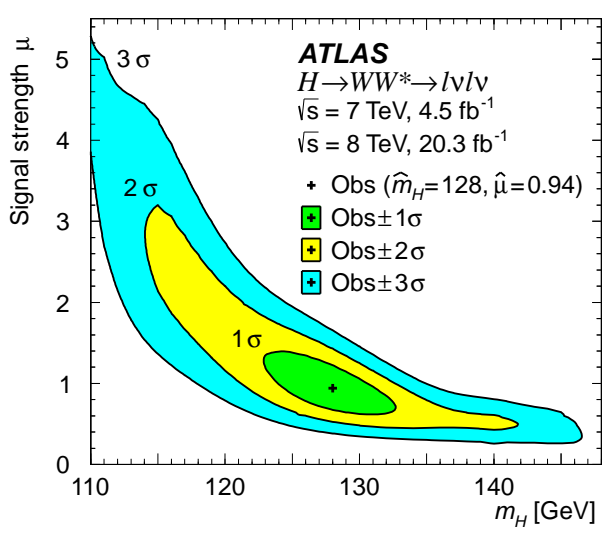

(b)

Figure 6: (a) Post-fit combined transverse mass distributions for $n_{j} \leq 1$ for all lepton-flavor samples. The bottom plot shows the residuals of the data with respect to the estimated background compared to the expected distribution for a SM Higgs boson with $m_{H}=125 \mathrm{GeV}$; the error bars on the data are statistical. The uncertainty on the background (shown as the shaded band around 0 ) is partially correlated between bins. (b) Observed signal strength $\mu$ as a function of $m_{H}$ as evaluated by the likelihood fit. The shaded areas represent the one, two, and three standard deviation contours with respect to the best fit values [27].

\section{$5.4 H \rightarrow Z \gamma \rightarrow \ell \ell \gamma(\ell=e$ or $\mu)$ decay channel}

This is a rare but sensitive to new physics decay mode. The branching ratio of $H \rightarrow Z \gamma$ is similar to the $H \rightarrow \gamma \gamma$, but the branching ratio of $Z$ to leptons leads to a rate which is only $5 \%$ compared to the $H \rightarrow \gamma \gamma$ one and leads to an expected signal to background ratio of $1 \%$. The dominant irreducible background is due to $Z \gamma$ continuum production (82\%). The $Z$ plus jets production has significant contribution $(17 \%)$ to the reducible background, while other sources 
have very little contribution. The signal is two opposite sign same flavor isolated leptons close to the $Z$ mass plus one isolated high $p_{\mathrm{T}}$ photon. The measurement is statistically limited and no excess of events over the SM prediction is seen. A 95\% CL limit is therefore set, which at $m_{H}=125.5 \mathrm{GeV}$ is 11 times the SM prediction (with 9 expected) [28].

\section{Search in fermionic decay modes}

Since the Higgs boson coupling is predicted to be proportional to mass of the quarks, the decay $H \rightarrow t \bar{t}$ should have been the one with the highest BR. However, this decay is not kinematically allowed for $m_{H}=125 \mathrm{GeV}$. Thus, the $H \rightarrow b \bar{b}$ decay has the highest BR ( $58 \%$ ) and the only way to probe the Higgs boson top quark Yukawa coupling, is through its associated production $t \bar{t} H$. The $H \rightarrow b \bar{b}$ final state suffers from huge QCD multijet backgrounds, making impossible the tagging of this final state alone. The only feasible way for this measurement, is through the use of associated production with a vector boson or a $t \bar{t}$ pair. The smaller rate of these processes in the presence of -still- large background makes their detection challenging. More favorable signal-to-background conditions are expected for $H \rightarrow \tau^{+} \tau^{-}$decays.

\section{1 $H \rightarrow \tau^{+} \tau^{-}$decay channel}

In this analysis [29], all combinations of leptonic $(\tau \rightarrow \ell v \bar{v}$ with $\ell=e, \mu)$ and hadronic $(\tau \rightarrow$ hadrons plus $v$ ) tau decays are considered. Therefore, three analysis channels denoted as $\tau_{\text {lep }} \tau_{\text {lep }}$, $\tau_{\text {lep }} \tau_{\text {had }}$ and $\tau_{\text {had }} \tau_{\text {had }}$ are investigated. Single lepton, dilepton and di- $\tau_{\text {had }}$ triggers were used to select the events. Electrons and muons are identified as described in sections 4.1 and 4.2 and required to have $p_{\mathrm{T}}$ above 15 and $10 \mathrm{GeV}$ respectively. Hadronic $\tau$ candidates are reconstructed as described in section 4.4 and are required to have $p_{\mathrm{T}}>25 \mathrm{GeV}$ and $\eta<2.47$. Tighter kinematic requirements are applied according to the sub-channel and the trigger used to select the events. The invariant $\tau \tau$ mass, $m_{\tau \tau}^{M M C}$, is reconstructed using the missing mass calculator [30].

The irreducible background consists of the much more copiously produced $Z \rightarrow \tau^{+} \tau^{-}$decay. Data driven techniques using $Z \rightarrow \mu^{+} \mu^{-}$events with the $\mu$ 's replaced by $\tau$ 's, are used to model this background. Depending on the sub-channel topology, several reducible backgrounds occur, such as QCD multijets, $W$ plus jets, diboson, $t \bar{t}$ and single top production, which are also estimated by data driven methods. Further categorization is applied to exploit the final states from different production modes of the Higgs boson to increase the sensitivity of the search. Two dedicated categories are considered to achieve both a high signal-to-background ratio and a good resolution for the reconstructed $\tau \tau$ invariant mass, using BDTs. The VBF category, enriched in events produced via vector-boson fusion, is defined by the presence of two jets with a large separation in pseudorapidity. The boosted category contains events where the reconstructed Higgs boson candidate has a large transverse momentum. It is dominated by events produced via ggF with additional jets from gluon radiation, but it also targets the $\mathrm{VH}$ associated production of the Higgs boson.

The major experimental systematic uncertainties result from uncertainties on efficiencies for triggering, object reconstruction and identification, as well as from uncertainties on the energy scale and resolution of jets, hadronically decaying taus and leptons. In general, the effects resulting from lepton-related uncertainties are smaller than those from jets and taus. Additional significant contribution to the uncertainty stems from the data driven evaluation of the backgrounds. 
The BDT output in the six analysis categories provides the final discrimination between signal and background. A maximum-likelihood fit is performed on all categories simultaneously to extract the signal strength. The likelihood is maximized on the BDT distributions in the signal regions, with information from control regions included to constrain background normalisations. The observed siginificance of the excess of events is $4.5 \sigma$ (with $3.4 \sigma$ expected). The final result of the Run I analysis for the combined signal strength measurement for $H \rightarrow \tau^{+} \tau^{-}$yields $\mu=1.43_{-0.26}^{+0.27}$ (stat) ${ }_{-0.25}^{+0.32}$ (syst) \pm 0.09 (theory). Figure 7 (a) shows the expected and observed number of events, in bins of $\log _{10}(\mathrm{~S} / \mathrm{B})$, for all signal region bins. Here, S/B is the signal-to-background ratio calculated assuming $\mu=1.4$ for each BDT bin in the signal regions. The expected signal yield for both $\mu=1$ and the best-fit value $\mu=1.4$ for $m_{H}=125 \mathrm{GeV}$ is shown on top of the background prediction from the best-fit values. The background expectation where the signal-strength parameter is fixed to $\mu=0$ is also shown for comparison. To visualise the compatibility of this excess of events above background predictions with the SM Higgs boson at $m_{H}=125 \mathrm{GeV}$, a weighted distribution of events as a function of $m_{\tau \tau}^{M M C}$ is shown in figure $7(\mathrm{~b})$. The events are weighted by a factor of $\log (1+\mathrm{S} / \mathrm{B})$, which enhances the events compatible with the signal hypothesis. Three hypotheses for the SM Higgs boson mass are shown. The excess of events in this mass distribution is consistent with the expectation for a Standard Model Higgs boson with $m_{H}=125 \mathrm{GeV}$, while it is less compatible with the other masses considered.

\section{2 $V H \rightarrow V b \bar{b}$ decay channel}

In this analysis [31] two b-tagged jets are required as well as the signature of a leptonic decay of a vector boson, namely $Z \rightarrow v v, W \rightarrow \ell v$ and $Z \rightarrow \ell^{+} \ell^{-}$, where $(\ell=e, \mu)$. The main components of the background are $W / Z$ plus jets, single top, $t \bar{t}$, diboson production and QCD multijet production. Single-lepton and dilepton triggers are used to select events with a least one lepton. In addition $E_{\mathrm{T}}^{\mathrm{miss}}$ triggers are used to select events in the 0-lepton category and increase the acceptance in the 1-lepton category. A $p_{\mathrm{T}}$ threshold of $20 \mathrm{GeV}$ is applied to all jets. Only events with two or three jets are selected. The data are divided in bins of $p_{\mathrm{T}}$ of the vector boson, number of leptons, number of jets and number of b-jets. Additional topological and kinematic criteria are applied to reject background events and enhance the sensitivity of the search in the different categories. Two parallel analyses are performed. The first is a cut-based analysis using as discriminant the invariant mass of the two b-tagged jets and the second a multivariate analysis using BDTs. Control regions are formed to control both the shape and normalization of different components of the background, while the QCD multi-jet background is completely estimated using data driven methods. A likelihood fit is used to simultaneously extract both the signal yield and constraints on the background normalisations and shapes. The distributions used by the fit are those of the dijet mass or the BDT discriminant.

A binned likelihood function is constructed as the product of Poisson-probability terms over the bins of the input distributions involving the numbers of data events and the expected signal and background yields, taking into account the effects of the floating background normalisations and the systematic uncertainties.

Diboson production with a $Z$ boson decaying to a pair of b-quarks and produced in association with either a $W$ or $Z$ boson has a signature very similar to the one considered in this analysis, but with a $m_{b b}$ distribution peaking at lower values. The cross section is about five times larger 


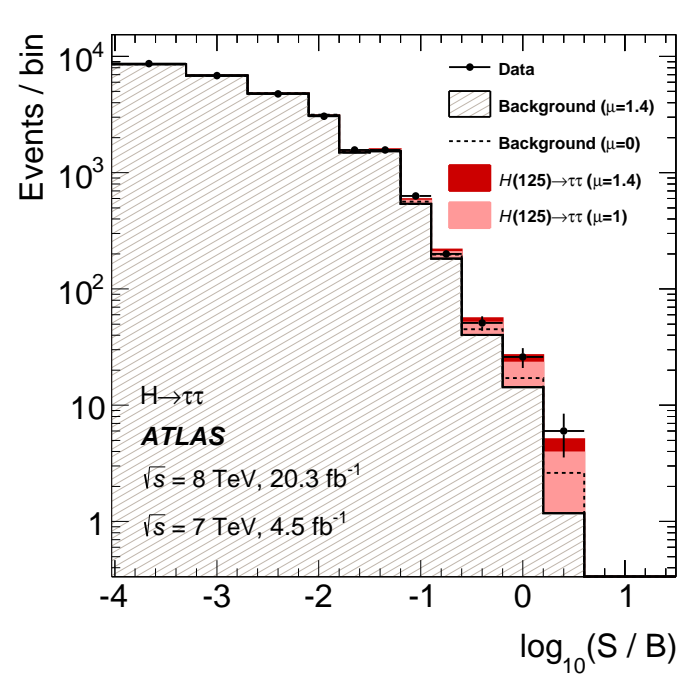

(a)

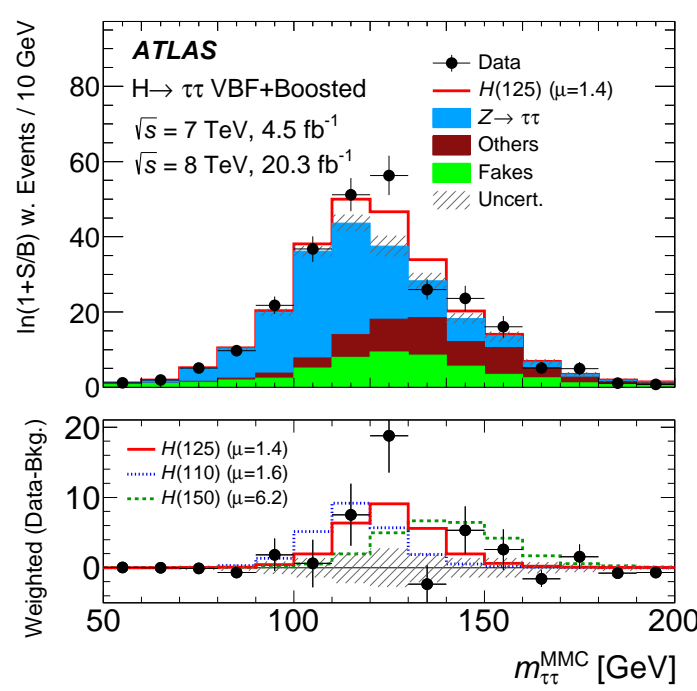

(b)

Figure 7: (a) Event yields as a function of $\log _{10}(\mathrm{~S} / \mathrm{B})$, where $\mathrm{S}$ (signal yield) and B (background yield) are taken from the BDT output bin of each event, assuming a signal strength $\mu=1.4$. Events in all categories are included. The predicted background is obtained from the global fit (with $\mu=1.4$ ), and signal yields are shown for $m_{H}=125 \mathrm{GeV}$ at $\mu=1$ and $\mu=1.4$ (the best-fit value). The background-only distribution (dashed line) is obtained from the global fit, with $\mu$ fixed at zero. (b) Distribution of the reconstructed invariant $\tau \tau$ mass, where events are weighted by $\log (1+\mathrm{S} / \mathrm{B})$ for all channels. The bottom panel shows the difference between weighted data events and weighted background events (black points), compared to the weighted signal yields. The $m_{H}=125 \mathrm{GeV}$ signal is plotted with a solid red line, and, for comparison, signals for $m_{H}$ $=110 \mathrm{GeV}$ (blue) and $m_{H}=150 \mathrm{GeV}$ (green) are also shown. The signal strengths of the best-fit values are used [29].

than for the SM Higgs boson with a mass of $125 \mathrm{GeV}$. Diboson production is therefore used as a validation of the analysis procedure. The measured signal strength for the $8 \mathrm{TeV}$ dataset with the MVA approach is $\mu_{V Z}=0.77 \pm 0.10$ (stat.) \pm 0.15 (syst.), consistent with the SM expectation.

For a Higgs boson with a mass of $125.36 \mathrm{GeV}$, as measured by ATLAS (section 7.1), the signal strength for $V H \rightarrow V b \bar{b}$ is estimated as $\mu=0.52 \pm 0.32$ (stat) \pm 0.24 (syst) and corresponds to a deviation from the backgound-only hypothesis with observed significance $1.4 \sigma$ (with $2.6 \sigma$ expected). As is shown in figure $8(\mathrm{a})$, the probability $p_{0}$ of obtaining from background alone a result at least as signal like as the observation is $8 \%$ for a tested Higgs boson mass of $125 \mathrm{GeV}$; in the presence of a Higgs boson with that mass and the SM signal strength, the expected $p_{0}$ value is $0.5 \%$. Figure $8(\mathrm{~b})$ shows the data, background and signal yields, where the final-discriminant bins in all signal regions are combined into bins of $\log (\mathrm{S} / \mathrm{B})$, separately for the $8 \mathrm{TeV}$ datasets, where $\mathrm{S}$ is the expected signal yield and B is the fitted background yield.

\section{3 $H \rightarrow \mu^{+} \mu^{-}$decay channel}

Since the Higgs boson coupling to fermions is predicted to be proportional to the mass of the corresponding fermions, the branching ratio for this decay is very small $\sim 2 \cdot 10^{-4}$. The signal to background ratio is small as well $\sim 0.2 \%$, but the dimuon spectrum provides a very clean signature 


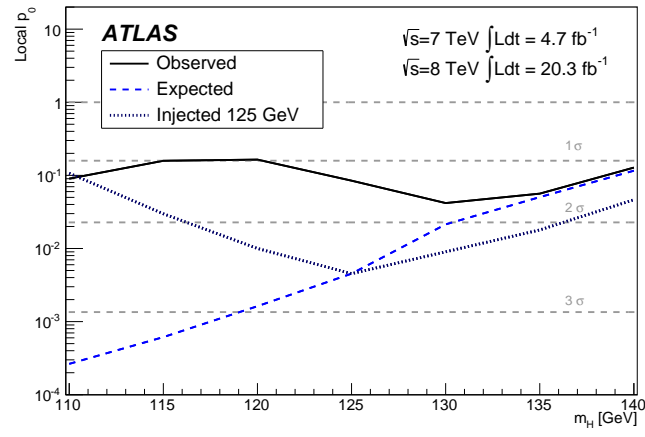

(a)

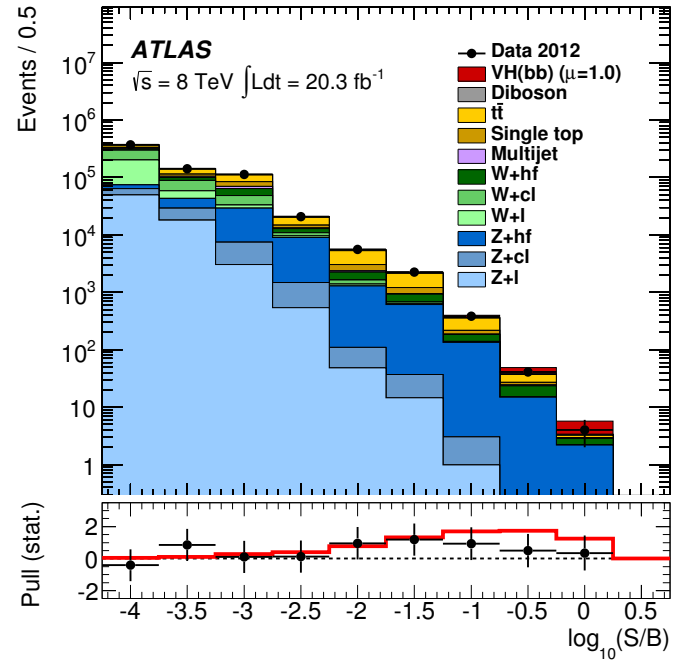

(b)

Figure 8: (a) Observed (solid) and expected $p_{0}$ values as a function of $m_{H}$ for all channels and data-taking periods combined. The expected $p_{0}$ values are given for the background-only hypothesis in the presence of a SM Higgs boson: for the dashed curve the Higgs boson mass corresponds to each tested mass point in turn; for the dotted curve the Higgs boson mass is $125 \mathrm{GeV}$. (b) Event yields as a function of $\log (\mathrm{S} / \mathrm{B})$ for data, background and Higgs boson signal with $m_{H}=125 \mathrm{GeV}$ for the $8 \mathrm{TeV}$ data. Final-discriminant bins in all signal regions are combined into bins of $\log (\mathrm{S} / \mathrm{B})$. The signal $\mathrm{S}$ and background $\mathrm{B}$ yields are the expected and the fitted ones, respectively. The Higgs boson signal contribution is shown as expected for the SM cross section (indicated as $\mu=1.0$ ). The pull of the data with respect to the background-only prediction is also shown with statistical uncertainties only. The full line indicates the pull of the prediction for the signal with $\mu=1.0[31]$.

with a very good mass resolution and at the same time is the only means of measuring the second generation fermionic couplings. The signal is exactly two isolated opposite sign muons, while there is a huge irreducible background from Drell-Yan. A cut-based analysis is used in ATLAS [32] using analytical modeling for the signal and background. The data are separated into the ggF and VBF categories. The main source of systematics comes from theory. No excess of events is observed, leading to an observed limit at 95\% CL, of 7.0 times the SM production rate at a Higgs boson mass of $125.5 \mathrm{GeV}$ (with 7.2 expected).

\section{Higgs properties}

Immediately after the discovery, the ATLAS collaboration has put great effort to study the properties of the newly discovered boson. Since the only free parameter in SM is its mass, this parameter was the first that had to be determined. In order to verify the SM origin or not of the Higgs boson, detailed measurements of its couplings, spin, parity and width had to be performed.

\subsection{Mass}

In the SM, the Higgs boson mass is not predicted. Its measurement is therefore required 
for precise calculations of electroweak observables including the production and decay properties of the Higgs boson itself. The two channels that are most sensitive to the Higgs boson mass measurement are the $H \rightarrow \gamma \gamma$ and $H \rightarrow Z Z^{*} \rightarrow 4 \ell$. The Higgs boson produces a narrow mass peak with a typical experimental resolution of $1.6 \mathrm{GeV}$ to $2 \mathrm{GeV}$ over a smooth background. The event selection for these channels is described in sections 5.1 and 5.2. As it was discussed earlier (see sections 4.1 and 4.2), several improvements on the energy (momentum) scale uncertainties of photons, electron and muons have been incorporated in the final ATLAS analysis [13] of Run I data, that affect directly the determination of the Higgs boson mass.

In the $H \rightarrow \gamma \gamma$ channel, the signal mass spectrum is modeled by the sum of a Crystal Ball function for the bulk of the events, which have a narrow Gaussian spectrum in the peak and tails toward lower reconstructed mass, and a wide Gaussian distribution to model the far outliers in the mass resolution. The parameters of the Crystal Ball and Gaussian functions, and their dependence on the Higgs boson mass, are fixed by fits to simulation samples at discrete mass values to obtain a smooth signal model depending only on the assumed Higgs boson mass and yield. The accuracy of this procedure is checked by fitting the Higgs boson mass in simulated samples with this signal model and is found to be better than $0.01 \%$ of the Higgs boson mass. The background is obtained directly from a fit to the diphoton mass distribution in the data over the range $105-160 \mathrm{GeV}$ after final selection. The mass spectra for the ten data categories and the two center-of-mass energies are fitted simultaneously using an unbinned maximum likelihood fit. The measured Higgs boson mass in the $H \rightarrow \gamma \gamma$ channel is [13]:

$$
m_{H}=125.98 \pm 0.42 \text { (stat) } \pm 0.28 \text { (syst) } \mathrm{GeV}
$$

Several methods are used to measure the Higgs boson mass in the $H \rightarrow Z Z^{*} \rightarrow 4 \ell$ decay channel. The two dimensional fit to the $m_{4 \ell}$ and the $B D T_{Z Z}$ output is chosen as the baseline method. The signal model is based on simulation distributions that are smoothed using a kernel density estimation method [33]. These distributions are generated at 15 different $m_{H}$ values in the mass range between 115 and $130 \mathrm{GeV}$ and form templates that are parameterized as a function of $m_{H}$. The background model is described using a full two dimensional PDF that is derived from simulation for the $Z Z$ background and by using data-driven techniques for the reducible background. The mass spectra for the eight data categories are fitted simultaneously using an unbinned maximum likelihood fit. The measured Higgs boson mass in the $H \rightarrow Z Z^{*} \rightarrow 4 \ell$ channel is [13]:

$$
m_{H}=124.51 \pm 0.52 \text { (stat) } \pm 0.06 \text { (syst) } \mathrm{GeV}
$$

For the combined mass measurement, hypothesized values of $m_{H}$ are tested using the profile likelihood ratio defined in terms of $m_{H}$ and treating $\mu_{\gamma \gamma}\left(m_{H}\right)$ and $\mu_{4 \ell}\left(m_{H}\right)$ as independent nuisance parameters, so as to make no assumptions about the SM Higgs couplings. The leading source of systematic uncertainty on the mass measurement comes from the energy and momentum scale uncertainties on the main physics objects used in the two analyses, namely photons for the $H \rightarrow \gamma \gamma$ and muons and electrons and muons for the $H \rightarrow Z Z^{*} \rightarrow 4 \ell$ final state. The correlation between the two measurements stems from common systematic uncertainties of the photon and the electron energy scale and is modeled in the combination by correlating the corresponding nuisance parameters. The combined mass measurement result is [13]: 


$$
m_{H}=125.36 \pm 0.37 \text { (stat) } \pm 0.18 \text { (syst) } \mathrm{GeV}
$$

To directly quantify the level of consistency between the individual measurements of the Higgs boson mass in the two channels, the profile likelihood used for the mass combination is parameterized as a function of the difference in measured mass values with the common mass $m_{H}$ profiled in the fit. A compatibility of $4.9 \%$, corresponding to $1.97 \sigma$, is obtained.

\subsection{Couplings}

In this section, the combined analyses of the Higgs boson production and decay rates as well as its coupling strengths to vector bosons and fermions are presented. The combinations take inputs from all the analyses presented in the previous sections, as well as the constraints on the ttH Higgs boson production [34-36] and recent results from [37] on the $V H \rightarrow V W W$ component, which are not included in this document. The statistical treatment of the data is described in Refs. [3842]. Hypothesis testing and confidence intervals are based on the profile likelihood ratio [43] test statistic. Figure 9 shows the updated measurements of the signal-strength parameter from a simultaneous fit to all decay channels that were analysed. The measurements are consistent and compatible with a single value with a probability of $76 \%$. Assuming a common multiplier to all signal yields, they can be combined to result in a global signal-strength value of [44]:

$$
\mu=1.18_{-0.14}^{+0.15}=1.18 \pm 0.10(\text { stat }) \pm 0.07(\text { expt })_{-0.07}^{+0.08}(\text { theo })
$$

consistent with the SM expectation of $\mu=1$ with a probability of $18 \%$. The uncertainty on the combination has comparable statistical and systematic components. The theoretical uncertainty includes contributions from SM cross sections and branching ratios as well as on the modeling of the production modes and the decays of the Higgs boson. Theoretical uncertainties on background processes are included in the uncertainty labeled as experimental systematic uncertainty. A significant component of the theoretical uncertainty is associated with the SM predictions of the Higgs boson production cross sections and decay branching ratios. Advances in theoretical calculations are required to improve the precision of future measurements.

The measurement of the signal strength described above, assumes that the relative contributions of different Higgs boson production processes and/or decay channels are equal to the SM predictions. Thus they may conceal differences between data and theory. Therefore, in addition to the signal strengths of different decay channels, the signal strengths of different production modes are determined, exploiting the sensitivity offered by the use of event categories in the analyses of all channels. The Higgs boson production processes can be categorised into two groups according to the Higgs boson couplings to fermions ( $g g F$ and ttH) or vector bosons (VBF and $\mathrm{VH}$ ). Potential deviations from the SM can be tested with two signal-strength parameters. The relative production cross sections of the vector boson and fermion-mediated processes can be tested using the ratio of the corresponding signal strengths. Figure 10(a) shows this ratio when it is measured separately for each decay channel. The combination of these measurements yields an overall value of the cross section ratio between the boson- and fermion-mediated processes (relative to its SM prediction) [44]:

$$
R=0.96_{-0.31}^{+0.43}=0.96_{-0.26}^{+0.34}(\text { stat })_{-0.13}^{+0.19}(\operatorname{expt})_{-0.10}^{+0.18}(\text { theo })
$$




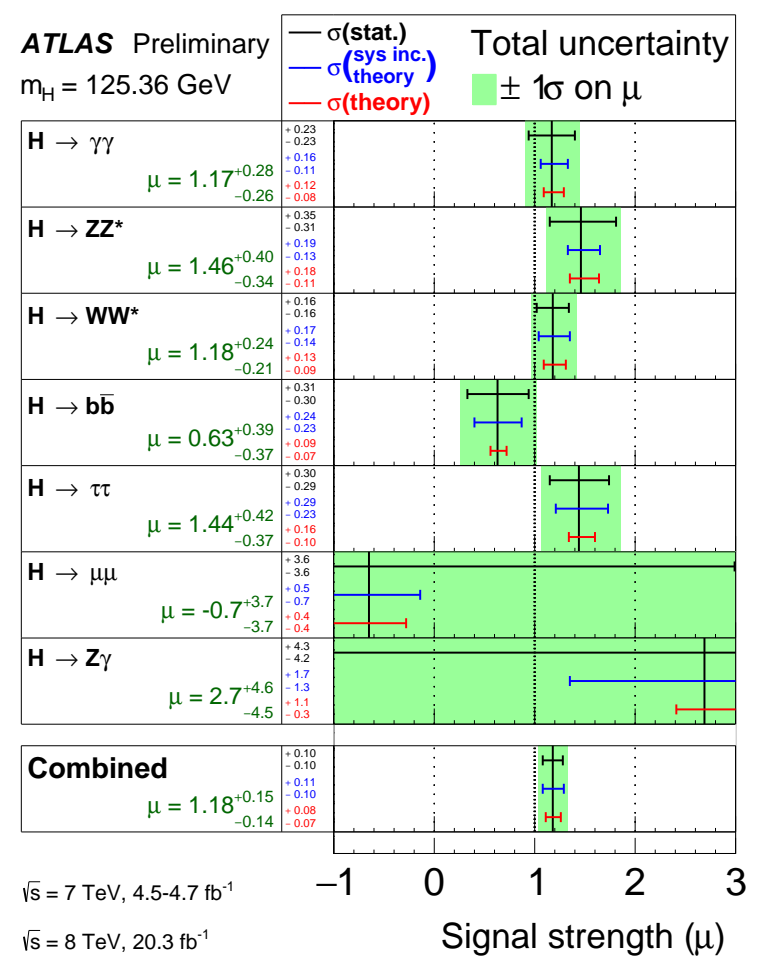

Figure 9: The observed signal strengths and uncertainties for different Higgs boson decay channels and their combination for $m_{H}=125.36 \mathrm{GeV}$. Higgs boson signals corresponding to the same decay channel are combined together for all analyses. The best-fit values are shown by the solid vertical lines. The total $\pm \sigma$ uncertainties are indicated by green shaded bands, with the individual contributions from the statistical uncertainty (top), the total (experimental and theoretical) systematic uncertainty (middle), and the theory systematic uncertainty (bottom) on the signal strength shown as horizontal error bars [44].

consistent with the SM expectation of one.

The Higgs boson production modes can be probed with four different signal-strength parameters: ggF, VBF, VH and ttH -one for each main production mode- assuming the SM values for the Higgs boson decay branching ratios. The SM predictions of the signal yields are scaled by these four production-dependent parameters. Their combined fitted values are illustrated in Figure 10(b). The signal strength measurements are in reasonable agreement with the SM predictions of unity. The significance for each process is calculated from a likelihood scan while contributions from other processes are profiled. The result provides strong evidence at the $4.3 \sigma$ level of the vector boson fusion production of the Higgs boson and supports the SM assumptions of production in association with vector bosons or a pair of top quarks.

In these results the signal strength scale factors for the given Higgs boson production or decay modes are used. However, for a measurement of Higgs boson coupling strengths, the production and decay modes cannot be treated independently, since each observed process involves at least two Higgs boson coupling strengths. Scenarios with a consistent treatment of coupling strengths in production and decay modes are studied in publication [44], but are beyond the scope of this document. However it is worth mentioning a result of these studies, which provides $\sim 4.5 \sigma$ evidence 


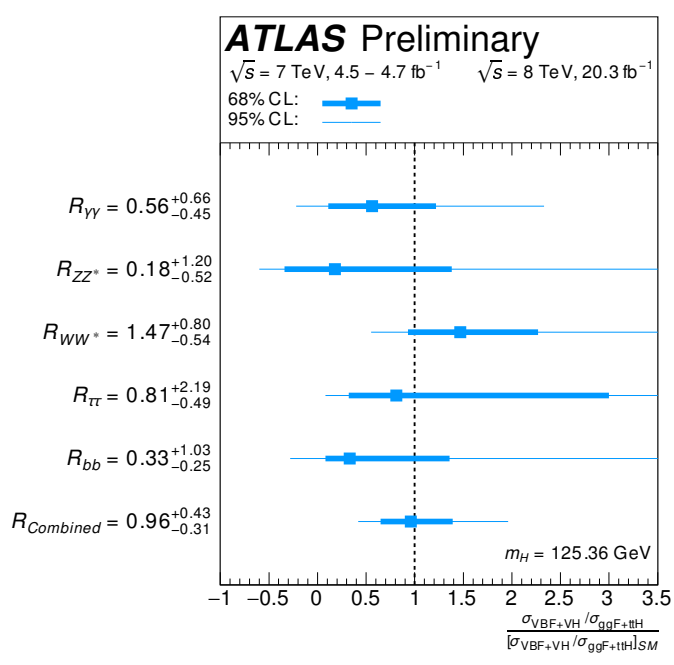

(a)

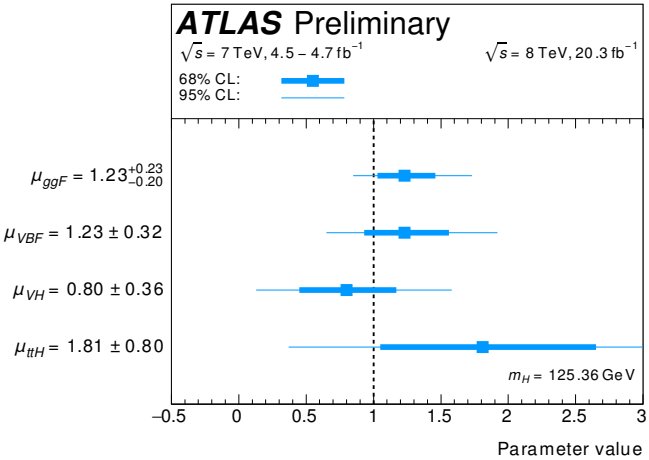

(b)

Figure 10: (a) The cross section ratios between vector boson and fermion-mediated processes relative to their SM values at $m_{H}=125.36 \mathrm{GeV}$, measured in the individual Higgs boson decay final states and their combination. (b) The best-fit signal-strength values of different production modes determined from the combined fit to the data. The inner and outer error bars represent 68\% CL and 95\% CL intervals, combining statistical and systematic uncertainties [44].

of the coupling of the Higgs boson to down-type fermions.

\subsection{Spin and Parity}

In the SM, the Higgs boson is a CP-even scalar particle, $J^{C P}=0^{++}$. Three possible scenarios for the spin and parity of the boson are considered: the hypothesis that the observed resonance is a spin-2 resonance, a pure spin-0 CP-even or CP-odd BSM Higgs boson, or a mixture of spin- 0 $\mathrm{CP}$-even and CP-odd states. The latter case would imply CP-violation in the Higgs sector. In the case of CP mixing, the Higgs boson would be a mass eigenstate, but not a CP eigenstate. In all cases, only one resonance with a mass of about $125 \mathrm{GeV}$ is considered. The study of the spin and parity properties of the Higgs boson in ATLAS is based on the $H \rightarrow \gamma \gamma, H \rightarrow Z Z^{*} \rightarrow 4 \ell$ and $H \rightarrow W W^{*} \rightarrow e v \mu \nu$ decay channels and their combination. It relies on discriminant observables chosen to be sensitive to the spin and parity of the signal while preserving the discrimination against the various backgrounds. Recent ATLAS studies [45] take advantage of improvements to the analysis strategy and to the modeling used to describe alternative spin hypotheses to improve previous ATLAS results [46].

Event selections for the $H \rightarrow Z Z^{*} \rightarrow 4 \ell$ and $H \rightarrow \gamma \gamma$ channels follow closely the ones described in sections 5.1 and 5.2. For the $H \rightarrow Z Z^{*} \rightarrow 4 \ell$ channel, events are further required to have a four lepton system invariant mass in the mass range between 115 and $130 \mathrm{GeV}$. The production and decay angles of the leptons are used as sensitive observables. Two approaches have been pursued to develop the discriminants to be used in order to distinguish between pairs of spin and parity states. The first uses the theoretical differential decay rate for the parity sensitive final state 
observables, corrected for the detector acceptance and analysis selection, to construct a matrix element based likelihood ratio analysis $\left(J^{P}\right.$-MELA), while the second approach is based on a BDT. Both analyses are complemented with a BDT discriminant designed to separate the signal from the $Z Z^{*}$ background. For the $H \rightarrow \gamma \gamma$ channel, events are further required to have a diphoton invariant mass in the mass range between 105 and $169 \mathrm{GeV}$. The kinematic variables sensitive to the spin of the resonance are the diphoton transverse momentum and the production angle of the two photons in the Collins-Soper frame [47]. Event selection of the spin and parity analysis in the $H \rightarrow W W^{*} \rightarrow e v \mu \nu$ channel follows the main aspects of the one presented in section 5.3 and is described in detail in publication [48]. The sensitive variables in this case are the mass, transverse momentum and azimuthal angular separation of the leptons, the transverse mass of the leptons and the missing transverse momentum. A BDT is used to construct the final discriminant.

A likelihood function that depends on the spin-parity assumption of the signal is constructed as a product of conditional probabilities over binned distributions of the discriminant observables in each channel. The test statistic used to distinguish between the two signal spin-parity hypotheses is based on a ratio of the corresponding profiled likelihoods. Using this framework, the SM Higgs boson hypothesis, corresponding to the quantum numbers $J^{P C}=0^{++}$, is tested against several alternative spin models. These include a non-SM spin- 0 and the spin- 2 model with universal and non-universal couplings to fermions and vector bosons. Some examples of distributions of the test statistic used to derive the results are presented in Figure 11. In these figures, the observed value is indicated by the vertical solid line and the expected medians by the dashed lines. The signal strengths per decay channel are treated as independent parameters during each fit. Their values are compatible with the SM predictions. The combination of the three decay processes allow the exclusion of all considered non-SM spin models at more then $99 \%$ CL in favour of the SM spin-0 hypothesis.

\subsection{Width}

Recent studies [49-52] have shown that the high-mass off-peak regions beyond $2 m_{V}$ ( $V=Z$ or $W$ ), the $H \rightarrow Z Z$ and $H \rightarrow W W$ channels are sensitive to Higgs boson production through off-shell and background interference effects. This is a novel way of characterising the properties of the Higgs boson in terms of the off-shell signal strength $\mu_{o f f-\text { shell }}$, and the associated off-shell Higgs boson couplings. This approach was used by the CMS Collaboration [53] to set an indirect limit on the total width and it is complementary to direct searches for Higgs boson to invisible decays, which will be presented in the next section.

The cross section for the off-shell Higgs boson production through gluon fusion with subsequent decay into vector-boson pairs is proportional to the product of the Higgs boson couplings squared for production and decay. However, unlike the on-shell Higgs boson production, it is independent of the total Higgs boson decay width $\Gamma_{H}$. The off-shell Higgs boson signal cannot be treated independently from the continuum $g g \rightarrow V V$ background, as sizeable negative interference effects appear [49]. The interference term is proportional to $\sqrt{\mu_{\text {off-shell }}}$. Assuming identical onshell and off-shell Higgs couplings, the ratio of $\mu_{o f f-\text { shell }}$ to $\mu_{\text {on-shell }}$ provides a measurement of the total width of the Higgs boson. The final ATLAS analysis [54] on Run I data is performed using the final states $Z Z \rightarrow 4 \ell, Z Z \rightarrow 2 \ell 2 v$ and $W W \rightarrow e v \mu v$. 


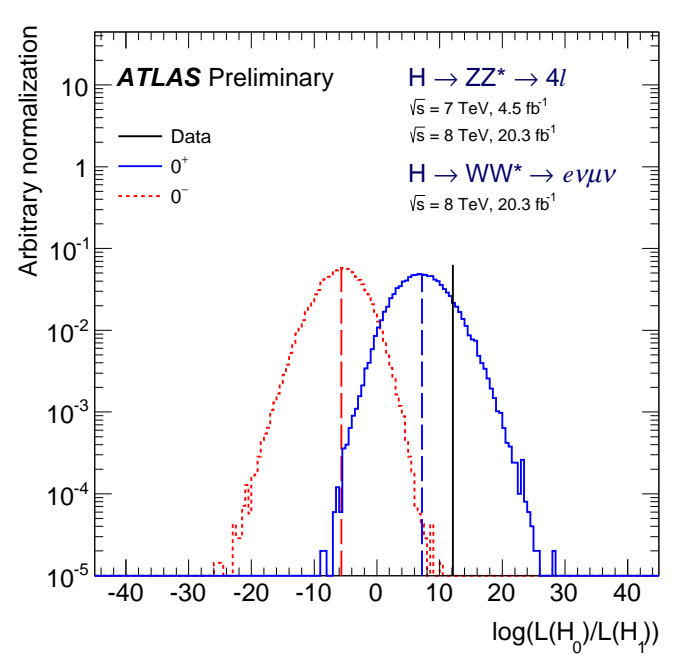

(a)

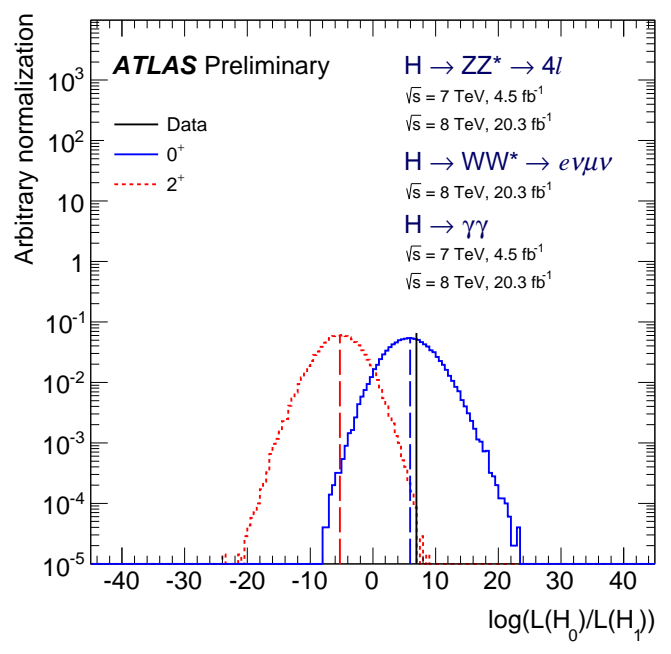

(c)

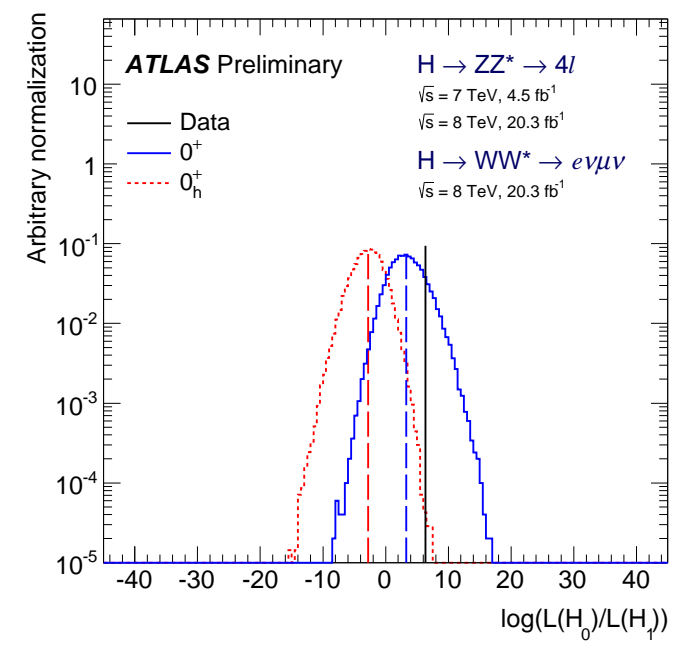

(b)

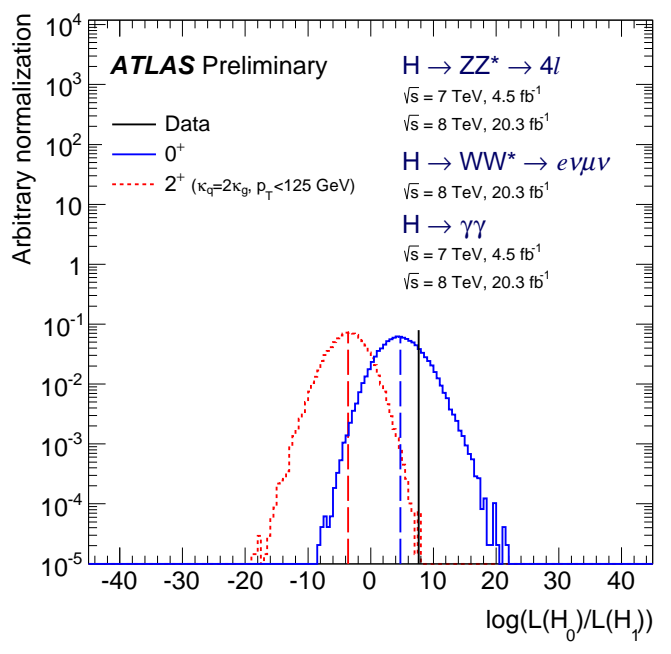

(d)

Figure 11: Examples of distributions of the test statistic for the combination of decay channels. (a) pseudoscalar hypothesis, (b) BSM scalar hypothesis, (c) spin-2 model with Universal couplings (d) spin-2 model with with low gluon fraction and $p_{\mathrm{T}}$ cut-off at $125 \mathrm{GeV}$ [45].

The event selection in the $H \rightarrow Z Z \rightarrow 4 \ell$ channel is exactly the same as the one presented in section 5.1. After having applied all the selection criteria, a matrix-element-based kinematic discriminant is formed to distinguish the signal from the background. Figure 12(a) shows the expected distribution of the matrix-element-based discriminant using $Z Z \rightarrow 4 \ell$ final state.

The analysis of the $H \rightarrow Z Z \rightarrow 2 \ell 2 v$ channel follows strategies similar to those used in the invisible Higgs boson search in the $Z H$ channel [56], with small differences in the optimization of the kinematic criteria. As the neutrinos in the final state do not allow a kinematic reconstruction of $m_{Z Z}$, the transverse mass $\left(m_{\mathrm{T}}^{Z Z}\right)$ is chosen as the discriminating variable to enhance sensitivity 


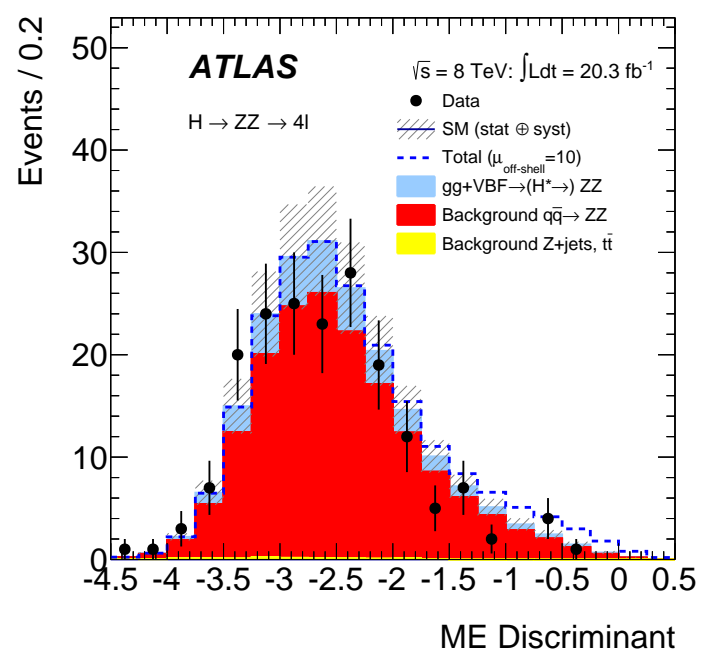

(a)

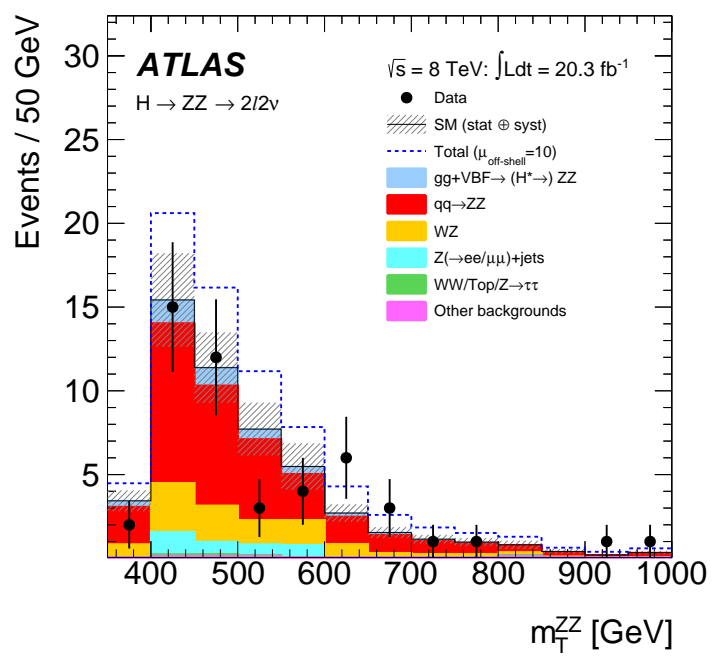

(b)

Figure 12: Observed distributions for (a) the ME-based discriminant for the $Z Z \rightarrow 4 \ell$ analysis and (b) the $Z Z$ transverse mass $m_{T}^{Z Z}$ for the $Z Z \rightarrow 2 \ell 2 v$ analysis; compared to the expected contributions from the SM including the Higgs boson (stack). The hatched area shows the combined statistical and systematic uncertainties. The dashed line corresponds to the total expected event yield, including all backgrounds and the Higgs boson with $\mu_{o f f-\text { shell }}=10$. A relative $g g \rightarrow Z Z$ background K-factor of 1 is assumed [54].

to the ggF $H \rightarrow Z Z$ signal. Figure 12(b) shows the observed distribution of $m_{\mathrm{T}}^{Z Z}$ compared to the predicted contributions from the SM.

The analysis of the $W W \rightarrow e v \mu v$ channel follows closely the one presented in section 5.3 in the oppositely charged electron-muon pair final state and ensures orthogonality with the $H \rightarrow$ $Z Z \rightarrow 2 \ell 2 v$ final state. In order to isolate the off-shell Higgs boson production while minimising the impact of higher-order QCD effects on $g g \rightarrow W W$ kinematics, a new variable, $R_{8}$, is introduced as discriminat variable, with $R_{8}=\sqrt{m_{\ell \ell}^{2}+\left(\alpha \cdot m_{\mathrm{T}}^{W W}\right)^{2}}$. Both the coefficient $\alpha=0.8$ and the requirement $R_{8}>450 \mathrm{GeV}$ are optimised for off-shell signal sensitivity while also rejecting on-shell Higgs boson events.

The largest systematic uncertainties for this analysis arise from theoretical uncertainties on the $g g F H^{*} \rightarrow V V$ signal process, the $g g / q \bar{q} \rightarrow V V$ background processes and the interference between the $g g \rightarrow V V$ signal and background processes. An uncertainty of 20-30\% due to higherorder QCD and EW corrections to the off-shell $g g \rightarrow H^{*} \rightarrow V V$ is used in this analysis. The corresponding PDF uncertainty is found to be 10-20\%. For the $g g \rightarrow V V$ background, higherorder QCD calculations are not available. The gluon-induced part of the signal K-factor is applied to the background as well and results are then given as a function of the unknown K-factor ratio $\left(R_{H^{*}}^{B}\right)$ between background and signal. Limits are produced when profiling the coupling scale factors associated with the on- and off-shell production under the assumption that the relevant Higgs boson couplings are independent of the energy scale of the Higgs production. An observed (expected) $95 \%$ CL upper limit on $\Gamma_{H} / \Gamma_{H}^{S M}$ in the range 4.5-7.5 (6.5-11.2) is found depending on $R_{H^{*}}^{B}$. Using the same assumptions and $R_{H^{*}}^{B}=1$, this translates into an observed (expected) $95 \% \mathrm{CL}$ 
upper limit on the Higgs boson total width of 22.7 (33.0) MeV.

\section{Search for invisible decays of the Higgs boson}

Astrophysical observations provide strong evidence for dark matter that could be explained by the existence of weakly interacting massive particles (see [55] and the references therein). The Higgs boson might decay to a component of the dark matter or long-lived unstable particles which do not interact with the detector, provided this decay is kinematically allowed. This is referred as an invisible decay of the Higgs boson. In this case additional information in the final state is needed to search for such decays.

A study [56] has been carried out to search for invisible decays of the Higgs boson, when this is produced in association with a $Z$ boson. This signature consists of two same flavor opposite sign leptons consistent with a $Z$ decay, accompanied by missing momentum from the invisible Higgs decay products. Kinematic requirements based on the fact that the $Z$ decay products are recoiling against missing momentum are applied. A jet veto is also applied to reduce the $Z$ plus jets background. The dominant irreducible background is the continuum $Z Z$ production, with smaller contribution from $W Z$.

Recently, a new search [57] for the Higgs boson produced in the VBF process and decaying invisibly, was performed in ATLAS. The selection of two jets with a large separation in pseudorapidity and large missing momentum leaves mainly two backgrounds, $Z(\rightarrow v v)$ plus jets and $W(\rightarrow \ell v)$ plus jets where the charged-lepton in the final state is not identified in the detector. Both of them, as well as the smaller contribution from QCD multijet production are estimated in datadriven ways using dedicated control regions. In this study two jets with $p_{\mathrm{T}}$ greater than $75 \mathrm{GeV}$ (for the leading) and $50 \mathrm{GeV}$ (for the subleading) are required, as well as $E_{\mathrm{T}}^{\text {miss }}>150 \mathrm{GeV}$. Events with additional objects such as b-jets, or lepton candidates are rejected. Further kinematic requirements are applied to increase the sensitivity of the search.

No excess of events is observed in either analysis and therefore, limits are set to the Higgs boson branching ratio to invisible decay products. For the discovered Higgs boson, an upper limit of $75 \%$ (62\% expected) at $95 \% \mathrm{CL}$ is set from the $\mathrm{ZH}$ search, while the corresponding limit from the VBF search is $29 \%$ ( $35 \%$ expected).

\section{Search for BSM Higgs-like bosons}

A very important ingredient in Higgs physics studies, is to understand if the discovered boson is the only Higgs boson, or there are more Higgs-like particles, the existence of which, would uncover physics beyond the the Standard Model (BSM). Using the data of Run I, several searches have been performed to investigate the existence of BSM Higgs-like particles. In the case of the two-Higgs-doublet model (2HDM) [58], the addition of a second Higgs doublet leads to five Higgs bosons after the electroweak symmetry breaking. In the case where the Higgs potential of the 2HDM is CP-conserving, the Higgs bosons -after electroweak symmetry breaking- are two CPeven ( $\mathrm{h}$ and $\mathrm{H})$, one $\mathrm{CP}$-odd $(\mathrm{A})$ and two charged $\left(\mathrm{H}^{ \pm}\right)$Higgs bosons. The phenomenology of such a model is very rich and depends on several free parameters. However, in general it is possible to accommodate in the model a Higgs boson -similar to the discovered one- in the model. 
The ATLAS collaboration has searched for evidence of the existence of all these additional bosons. In particular, a search for neutral Higgs bosons with enhanced couplings to down-type fermions decaying to a pair of $\tau^{+} \tau^{-}$is presented in publication [59]. Searches for the charged Higgs bosons decays $H^{ \pm} \rightarrow \tau \nu, H^{ \pm} \rightarrow W Z$ and $H^{ \pm} \rightarrow c s$, are presented in publications [60-62]. A search for a heavy CP-odd Higgs decaying to a $Z$ boson and a Higgs boson $(A \rightarrow Z h)$ is presented in publication [63]. Finally, several searches are performed with high sensitivity on a Higgs boson hypothesis with high mass and are presented in publications [64-66]. Final results on these studies will appear soon.

The result of all these searches performed up to the writing of this document, is that no excess of events with respect to the SM background is observed. Therefore, limits constraining the parameters of the BSM models have been set.

\section{Summary}

Using the LHC Run I data set, the ATLAS and CMS experiments have made the discovery of a scalar Higgs boson, with mass $m_{H}=125.09 \pm 0.24 \mathrm{GeV}$ [67]. After its discovery several precision studies were performed in the ATLAS experiment to investigate the properties of the newly discovered particle. The studies showed that it has a narrow width $\Gamma_{H}<23 \mathrm{MeV}$, its spin and $\mathrm{CP}$ properties are consistent with the $J^{C P}=0^{++}$hypothesis and all bosonic and fermionic coupling measurements are in agreement with the SM predictions within the current uncertainties. Furthermore, in the search for additional Higgs bosons no significant excess of events has been observed in the various analyses.

More data will be collected in the upcoming LHC Run II, where the center-of-mass energy will be raised to $13-14 \mathrm{TeV}$ and the total luminosity is expected to be increased by a factor 4-5 with respect to Run I. This will allow an improved determination of the Higgs boson properties and the Higgs sector in theories beyond the Standard Model will be further investigated.

\section{Acknowledgements}

This research has been co-financed by the European Union (European Social Fund - ESF) and Greek National funds through the Operational Program "Education and Lifelong Learning" of the National Strategic Reference Framework (NSRF) - Research Funding Program: THALES. Investing in knowledge society through the European Social Fund.

\section{References}

[1] L. Evans and P. Bryant (editors) 2008 JINST 3 S08001.

[2] F. Englert and R. Brout, Phys. Rev. Lett.13 (1964) 321.

[3] P. W. Higgs, Phys. Rev. Lett. 13 (1964) 508.

[4] ATLAS Collaboration, JINST 3 (2008) S08003.

[5] CMS Collaboration, JINST 3 (2008) S08004.

[6] ATLAS Collaboration, Phys. Lett. B 716 (2012) 1. 
[7] CMS Collaboration, Phys. Lett. B 716 (2012) 30.

[8] The LHC Higgs cross section working group, Handbook of LHC Higgs cross sections [arXiv:1101.0593], [arXiv:1201.3084],[arXiv:1307.1347].

[9] ATLAS Collaboration, Eur. Phys. J. C 72 (2012) 1909.

[10] ATLAS Collaboration, Eur. Phys. J. C 74 (2014) 3071.

[11] ATLAS Collaboration, ATLAS-CONF-2012-047 (2012), http://cds.cern.ch/record/1449796.

[12] ATLAS Collaboration, Phys. Rev. D 83 (2011) 052005.

[13] ATLAS collaboration, Phys. Rev. D 90 (2014) 052004.

[14] ATLAS Collaboration, Eur. Phys. J. C 74 (2014) 3130.

[15] M. Cacciari, G.P. Salam, and G. Soyez, J. High Energy Phys. 04 (2008) 063.

[16] ATLAS Collaboration, Eur. Phys. J. C 73 (2013) 2304.

[17] ATLAS Collaboration, Eur. Phys. J. C 75 (2015) 17.

[18] ATLAS Collaboration, ATLAS-CONF-2013-083 (2013), http://cds.cern.ch/record/1570994.

[19] ATLAS Collaboration, ATLAS-CONF-2011-102 (2011), http://cds.cern.ch/record/1369219.

[20] ATLAS Collaboration, ATLAS-CONF-2014-046 (2014), http://cds.cern.ch/record/1741020.

[21] P. Speckmayer, A. Hocker, J. Stelzer, and H. Voss, J. Phys. Conf. Ser. 219 (2010) 032057.

[22] ATLAS Collaboration, [arXiv:1412.7086], submitted to Eur. Phys. J. C.

[23] ATLAS Collaboration, Eur. Phys. J. C 72 (2012) 1844.

[24] ATLAS Collaboration, ATLAS-CONF-2012-143 (2012), http://cds.cern.ch/record/1491697.

[25] ATLAS Collaboration, Phys. Rev. D 91 (2015) 012006.

[26] ATLAS collaboration, Phys. Rev. D 90 (2014) 112015.

[27] ATLAS collaboration, [arXiv:1412.2641], submitted to Phys. Rev. D.

[28] ATLAS collaboration, Phys. Lett. B 732 (2014) 8.

[29] ATLAS collaboration, [arXiv:1501.4943], submitted to JHEP.

[30] A. Elagin et al., Nucl. Instrum. Meth. A 654 (2011) 481.

[31] ATLAS collaboration, JHEP 01 (2015), 069.

[32] ATLAS collaboration, Phys. Lett B 738 (2014) 68.

[33] K. S. Cranmer, Comput. Phys. Commun. 136 (2001) 198.

[34] ATLAS collaboration, Phys. Lett. B 740 (2015) 222.

[35] ATLAS collaboration, [arXiv:1503.5066], submitted to Eur. Phys. J. C.

[36] ATLAS collaboration, ATLAS-CONF-2015-006, http://cds.cern.ch/record/2002125.

[37] ATLAS collaboration, ATLAS-CONF-2015-005, http://cds.cern.ch/record/2002123.

[38] ATLAS Collaboration, Phys. Rev. D 86 (2012) 032003. 
[39] ATLAS and CMS Collaborations, ATL-PHYS-PUB-2011-011, CERN-CMS-NOTE-2011-005 (2011), http://cds.cern.ch/record/1379837.

[40] L. Moneta, et al., PoS ACAT2010 (2010) 057.

[41] K. Cranmer, G. Lewis, L. Moneta, A. Shibata, and W. Verkerke, CERN-OPEN-2012-016 (2012).

[42] W. Verkerke and D. Kirkby, arXiv:physics/0306116v1 [physics.data-an].

[43] G. Cowan, K. Cranmer, E. Gross, and O. Vitells, Eur. Phys. J. C 71 (2011) 1554.

[44] ATLAS collaboration, ATLAS-CONF-2015-007, http://cds.cern.ch/record/2002212.

[45] ATLAS Collaboration, ATLAS-CONF-2015-008 (2015), http://cds.cern.ch/record/2002414.

[46] ATLAS Collaboration, Phys. Lett. B 726 (2013) 120.

[47] J. C. Collins and D. E. Soper, Phys. Rev. D 16 (1977) 2219.

[48] ATLAS Collaboration, [arXiv:1503.3643], submitted to Eur. Phys. J. C.

[49] N. Kauer and G. Passarino, JHEP 08 (2012) 116.

[50] F. Caola and K. Melnikov, Phys. Rev. D 88 (2013) 054024.

[51] J. M. Campbell, R. K. Ellis, and C. Williams, JHEP 04 (2014) 060.

[52] J. M. Campbell, R. K. Ellis, and C. Williams, Phys. Rev. D 89 (2014) 053011.

[53] CMS Collaboration, Phys. Lett. B 736 (2014) 64.

[54] ATLAS collaboration, [arXiv:1503.1060], submitted to Eur. Phys. J. C.

[55] D. Clowe, et al, Astrophys.J. 648 (2006) L109.

[56] ATLAS collaboration, Phys. Rev. Lett. 112 (2014) 201802.

[57] ATLAS collaboration, ATLAS-CONF-2015-004, http://cds.cern.ch/record/2002121.

[58] G. Branco, et al., Phys. Rept. 516 (2012) 1.

[59] ATLAS collaboration, JHEP 11 (2014) 056.

[60] ATLAS collaboration, JHEP 03 (2015) 088.

[61] ATLAS collaboration, [arXiv:1503.4233], submitted to Phys. Rev. Lett.

[62] ATLAS collaboration, Eur. Phys. J. C 73 (2013) 2465.

[63] ATLAS collaboration, Phys. Lett. B 744 (2015) 163.

[64] ATLAS collaboration, Phys. Lett. B 717 (2012) 70.

[65] ATLAS collaboration, Phys. Lett. B 717 (2012) 29.

[66] ATLAS collaboration, Phys. Lett. B 718 (2012) 391.

[67] ATLAS and CMS Collaborations, [arXiv:1503.7589], submitted to Phys. Rev. Lett. 